\title{
The Roles of Resilience and Perceived Discrimination in Sexual Minority Identity Integration
}

\author{
Peter Alexander Brown \\ West Virginia University
}

Follow this and additional works at: https://researchrepository.wvu.edu/etd

\section{Recommended Citation}

Brown, Peter Alexander, "The Roles of Resilience and Perceived Discrimination in Sexual Minority Identity Integration" (2014). Graduate Theses, Dissertations, and Problem Reports. 343.

https://researchrepository.wvu.edu/etd/343

This Dissertation is protected by copyright and/or related rights. It has been brought to you by the The Research Repository @ WVU with permission from the rights-holder(s). You are free to use this Dissertation in any way that is permitted by the copyright and related rights legislation that applies to your use. For other uses you must obtain permission from the rights-holder(s) directly, unless additional rights are indicated by a Creative Commons license in the record and/ or on the work itself. This Dissertation has been accepted for inclusion in WVU Graduate Theses, Dissertations, and Problem Reports collection by an authorized administrator of The Research Repository @ WVU.

For more information, please contact researchrepository@mail.wvu.edu. 
The Roles of Resilience and Perceived Discrimination in

Sexual Minority Identity Integration

Peter Alexander Brown

Dissertation submitted to the College of Education and Human Services

at West Virginia University

in partial fulfillment of the requirements for the degree of

Doctor of Philosophy in

Counseling Psychology

\author{
Monica Leppma, Ph.D., Chair \\ James W. Bartee, Ph.D \\ Jeffrey Daniels, Ph.D. \\ Jennifer Taylor, Ph.D. \\ Dennis Allen, Ph.D.
}

Department of Counseling, Rehabilitation Counseling, and Counseling Psychology

Morgantown, West Virginia

2014

Keywords: LGB Identity, Sexual Minority Identity, Resilience, Discrimination Copyright 2014 Peter Alexander Brown 


\author{
ABSTRACT \\ The Roles of Resilience and Perceived Discrimination in \\ Sexual Minority Identity Integration
}

Peter Alexander Brown

\begin{abstract}
Although researchers and theorists have long moved away from public disclosure of sexual minority identity (i.e. coming out) as the theoretical apex of lesbian, gay, or bisexual (LGB) identity development, it is still considered an important process of overall identity integration. Unfortunately public disclosure of sexual minority identity may be dependent upon the social environment in which disclosure occurs. Given the variable nature of social contexts, public disclosure across domains of social functioning may be problematic. Previous research has suggested that disclosure is in part more or less likely to occur based on individual appraisal of discriminative experiences (Meyer, 2013). The purpose of the present study was to extend this research by establishing a moderating effect of resilience that may help buffer against the experience of discrimination in predicting identity integration. Respondents $(n=250)$ completed an online survey examining areas of outness, resilience, effect and frequency of perceived discrimination (EPD and FPD), and LGB identity status. Hierarchical regression results indicated that while resilience does not moderate EPD and FPD in predicting degree of outness, along with FPD it does independently predict outness. Additionally, post hoc regression analyses suggested variable predictive ability depending on demographic variables. Implications, limitations and future directions for these findings are discussed.
\end{abstract}


To Penelope and Adeline. This was all for you. 


\section{Acknowledgements}

First and foremost, I would like to thank my committee for their invaluable support and guidance through this process. To Dr. Monica Leppma, thank you for accepting the task of serving as my Chair, even when it was not your obligation or responsibility to do so. You have been incredibly supportive and encouraging, especially when the going got rough. It has been a privilege to be partnered with you on this project. To Dr. Jennifer Taylor, the circumstances under which you joined my team were not ideal at first, but I feel very fortunate that life gave me some lemons because this study became stronger and more interesting as a result of your participation. To Dr. Dennis Allen, I appreciate your initial encouragement and response to my ideas. I am also sincerely grateful for your participation, even though numbers were involved. To Dr. Jeffrey Daniels, thank you so much for your consistent guidance and scholarship for the three years that I walked those halls. It has been pleasure working with you. And to Dr. James Bartee, I would like to express my deepest possible gratitude for all of the unerring support and direction you have given me. Your mentorship and friendship have been invaluable to me in the truest sense of the word. The opportunity to learn from you is not what brought me to West Virginia, but it became one of the most important reasons to stay. Thank you for seeing in me a psychologist.

It would have not been possible for me to complete any of this without my friends and family, and so it is also not possible for me to complete it without thanking them. To my wonderful cohort, Joe, Kat, Narayan, Will, and Jes: we made it. I loved sharing this with all of you, and I wish you the best. Divide and conquer! To my friend Susan: Thank you for your smile, and thank you for mine. Love to Ryan, who was with me on 
that first rainy day in Portland, and whose passion rubbed off. To my incredible colleagues on internship, Brian, Rachel, Amber, and Lisa: One year was not nearly enough to be with you. We became psychologists together and I will never forget that. (And of course to the folks at Water Street Coffee, who will never know how much of this document was written right under their roof, thanks for the Tanzanian Peaberry.) To Mom, Dad, Mary, Drew, Cate, and Will, and to all of my unbelievably generous friends: thank you for understanding.

Finally, thank you to my wonderful wife and partner Meg, who always drew my attention to what was most important, and who looked for both of us when I could not see. I love you. 


\section{Table of Contents}

$\begin{array}{ll}\text { Abstract } & \text { ii }\end{array}$

Acknowledgements $\quad$ iv

Table of Contents $\quad$ vi

List of Tables $\quad$ ix

List of Figures $\quad$ X

List of Abbreviations $\quad$ xi

CHAPTER ONE: Introduction and Review of the Literature $\quad 1$

Statement of the Problem 2

Relevance to Counseling Psychology 5

$\begin{array}{ll}\text { Definition of Terms } & 8\end{array}$

Review of the Literature $\quad 11$

Sexual Minority Identity Development 11

$\begin{array}{ll}\text { Identity Integration } & 15\end{array}$

$\begin{array}{ll}\text { Perceived Discrimination } & 23\end{array}$

$\begin{array}{ll}\text { Resilience } & 27\end{array}$

$\begin{array}{ll}\text { Purpose Statement } & 32\end{array}$

$\begin{array}{ll}\text { Rationale } & 33\end{array}$

$\begin{array}{ll}\text { Research Questions } & 34\end{array}$

Research Question One $\quad 35$

Research Question Two 35

CHAPTER TWO: Methodology 36

Research Design 36 
$\begin{array}{ll}\text { Measures } & 38\end{array}$

The Outness Inventory $\quad 39$

The Courage to Challenge Scale 41

The Gay and Lesbian Oppressive Situations Inventory 42

The Lesbian, Gay, and Bisexual Identity Scale 43

$\begin{array}{ll}\text { Demographics } & 46\end{array}$

$\begin{array}{ll}\text { Procedure } & 46\end{array}$

$\begin{array}{ll}\text { Survey } & 46\end{array}$

$\begin{array}{ll}\text { Data Collection } & 48\end{array}$

$\begin{array}{ll}\text { Statistical Analysis } & 49\end{array}$

CHAPTER THREE: Results

Descriptive Statistics $\quad 54$

The Outness Inventory $\quad 54$

The Courage to Challenge Scale $\quad 54$

The Gay and Lesbian Oppressive Situations Inventory 55

The Lesbian, Gay, and Bisexual Identity Scale $\quad 55$

$\begin{array}{ll}\text { Analyses } & 56\end{array}$

$\begin{array}{ll}\text { Research Question } 1 & 56\end{array}$

$\begin{array}{ll}\text { Research Question } 2 & 58\end{array}$

Post Hoc Analyses $\quad 59$

CHAPTER FOUR: Discussion $\quad 63$

$\begin{array}{ll}\text { Findings and Implications } & 63\end{array}$ 
$\begin{array}{ll}\text { Research Question } 1 & 63\end{array}$

$\begin{array}{ll}\text { Research Question } 2 & 66\end{array}$

$\begin{array}{ll}\text { Post Hoc Analyses } & 69\end{array}$

$\begin{array}{ll}\text { Sexual Orientation } & 70\end{array}$

$\begin{array}{ll}\text { Gender } & 71\end{array}$

$\begin{array}{ll}\text { Age } & 72\end{array}$

$\begin{array}{ll}\text { Race/Ethnicity } & 73\end{array}$

$\begin{array}{ll}\text { Religion } & 74\end{array}$

$\begin{array}{ll}\text { Relationship Status } & 75\end{array}$

$\begin{array}{ll}\text { Education } & 76\end{array}$

$\begin{array}{ll}\text { Limitations } & 78\end{array}$

$\begin{array}{ll}\text { Future Directions } & 79\end{array}$

$\begin{array}{ll}\text { Conclusion } & 81\end{array}$

REFERENCES $\quad 83$

$\begin{array}{ll}\text { APPENDIX A: Demographic Questionnaire } & 106\end{array}$

$\begin{array}{ll}\text { APPENDIX B: Outness Inventory } & 108\end{array}$

APPENDIX C: Courage to Challenge Scale 109

APPENDIX D: Gay and Lesbian Oppressive Situations Inventory 111

APPENDIX E: Lesbian, Gay, and Bisexual Identity Scale $\quad 115$

APPENDIX F: Internal Review Board Approval (Initial) 117

APPENDIX G: Internal Review Board Approval (Amendment) 118

APPENDIX H: Informed Consent 119 


\section{List of Tables}

Table 1: Participant Demographics $\quad 94$

Table 2: Descriptive Statistics for All Instruments and Subscales 96

Table 3: Pearson Correlations for Outness 97

Table 4: Hierarchical Regression for Outness 98

Table 5: Forward Regression for Outness 99

Table 6: Backward Regression for Outness 100

Table 7: Pearson Correlations Between Predictor Variables and LGBIS Subscales 101

Table 8: Descriptive Statistics and Pearson Correlations for Outness by

$\begin{array}{ll}\text { Demographics } & 102\end{array}$

Table 9: Multiple Regressions for Outness by Demographics 103 


\section{List of Figures}

Figure 1: Proposed Role of Environmental Factors in Identity Integration 104

Figure 2: Proposed Moderating Effect of Resilience 


\section{List of Abbreviations}

\begin{tabular}{|c|c|}
\hline ATLGMS & Attitudes Towards Lesbians and Gay Men Scale \\
\hline CCS & Courage to Challenge Scale \\
\hline CDC & Centers for Disease Control and Prevention \\
\hline CI & Couples Issues (GALOSI subscale) \\
\hline DADT & Don’t Ask Don’t Tell \\
\hline $\mathrm{D} / \mathrm{C}$ & Deepening/Commitment \\
\hline DS & Danger to Safety (GALOSI subscale) \\
\hline EPD & Effect of Perceived Discrimination \\
\hline ERS & Exclusion, Rejection, and Separation (GALOSI subscale) \\
\hline FPD & Frequency of Perceived Discrimination \\
\hline GALOSI-E & Gay and Lesbian Oppressive Situations Inventory, Effect \\
\hline GALOSI-F & Gay and Lesbian Oppressive Situations Inventory, Frequency \\
\hline IH & Internalized Homonegativity (GALOSI subscale) \\
\hline $\mathbf{I} / \mathbf{S}$ & Internalization/Synthesis \\
\hline LGB & Lesbian, Gay, and Bisexual \\
\hline LGBIS & Lesbian, Gay, and Bisexual Identity Scale \\
\hline LGIS & Lesbian and Gay Identity Scale \\
\hline MHS & Modern Homonegativity Scale \\
\hline OGO & Other-group Orientation \\
\hline OI & Outness Inventory \\
\hline OTHI & Organized Tolerance for Heterosexism Inventory \\
\hline $\mathbf{R} \times \mathbf{E P D}$ & Denotes the statistical interaction between resilience and EP \\
\hline
\end{tabular}


$\mathbf{R} \times \mathbf{F P D} \quad$ Denotes the statistical interaction between resilience and FPD

ROR Restricted Opportunities and Rights (GALOSI subscale)

SDO Sexual Dominance Orientation

SGO Same-group Orientation

SS

SVS

VHI

WHEQ
Stigmatizing and Stereotyping (GALOSI subscale)

Structural Violence Scale

Verbal Harassment and Intimidation (GALOSI subscale)

Workplace Heterosexist Experiences Questionnaire 


\section{CHAPTER ONE: INTRODUCTION AND REVIEW OF THE LITERATURE}

In recent years, the lesbian, gay, and bisexual (LGB) community has been one of the most prominent minority groups in the United States. Due in part to widespread discourse regarding human rights and civil liberties, such as evolving access to equitable healthcare, armed service, and marriage, public opinion of this group has seen rapid change over the previous decade (Becker, 2014). Despite its high profile, however, population estimates for this group are inherently problematic. One widely reported figure suggests that 3.5-3.8\% of adults in the U.S. identify as LGB (Gates, 2011). Other findings, however, indicate that as much as twice that percentage $(4.4 \%$ of women; $6.2 \%$ of men) report being sexually attracted to members of the same gender (University of Chicago, 1994). Because sexuality is often understood to be a dynamic construct, rigid binary classifications (i.e. "gay" versus "straight") fail to capture many of the complex differences in orientation across individuals. For example, many who identify as LGB may not disclose their identity publicly, and would therefore be missed by such census attempts. Moreover, LGB individuals do not typically adopt sexual minority identity at birth or during childhood. Rather, this identity is usually accepted later in life, often beginning in adolescence or young-adulthood, with an extended process of identity development commonly associated with this event (Rosario, Schrimshaw, Hunter, \& Braun, 2006).

Consequently, the coming out process is often regarded as non-normative, not only by others but by the individuals themselves (Rosario et al., 2006). Because of this group's minority status, individuals who undergo LGB identity development may face significant challenges from their social environment, such as discrimination. Individuals 
may also find that they comfortable disclosing their minority identities in some social contexts more than others, which may be more or less accepting of their sexual orientation. Regardless of questions of when? and to whom?, it is critical to highlight that the decision to disclose one's identity status is not a singular or unique event; rather it is a continuous process. Individuals who decide to disclose their LGB identities may face the decision to do so on a regular basis and across the lifespan, with new acquaintances and in new settings (Legate, Ryan, \& Weinstein, 2012). Furthermore, the coming out process may be "an interpersonal skill that develops over time as GLB individuals gain experience as sexual minorities in a heterosexist, homophobic society," (Knoble \& Linville, 2012, p. 336).

Because stress associated with LGB identity development varies significantly across individuals, it has been hypothesized that different protective factors may be in place which help buffer against stress and promote resilience (Saewyc, 2011). Although much of the available literature has been concerned with external factors of resilience (e.g., non-discrimination policies in the workplace; Waldo, 1999), less is known about internal characteristics that may help to buffer against minority stress and foster healthy identity development. Resilience has been implicated in helping to facilitate minority identity development within the context of racial (Miller \& MacIntosh, 1999) and ethnic (Costigan, Koryzma, Hua, \& Chance, 2010) identities, with their role in sexual minority identity development receiving less attention in the literature (Bowleg et al., 2003).

\section{Statement of the Problem}

The construct of LGB identity as a developmental process has existed prominently in the literature since Cass (1979) introduced her influential six-stage model. 
The hallmark of this and subsequent models (e.g., D’Augelli, 1994; Troido, 1988) is the rejection of the assumption that LGB identity development is a single-event process. Prior to this, the disclosure of one's LGB identity to others, commonly termed "coming out", was considered to be the only stage of development. The coming out process remains integral to many models of LGB identity development, but it is usually recognized that this occurs only after "a series of complex cognitive, affective, and behavioral changes," (Reynolds \& Hanjorgiris, 2000, p. 37). Among the recent criticisms of the continued emphasis on coming out as a marker for LGB identity development, however, is the emphasis on public disclosure as a developmental milestone (Reynolds \& Hanjorgiris). This emphasis ignores cultural factors such as race/ethnicity, socioeconomic status (SES), and religion, as well as such environmental factors as career or work-setting and geographic location. The threat of discrimination following coming out is potentially severe (Fassinger \& Miller, 1996). The expectation that this act completely defines one's identity, therefore, is arguably unrealistic.

Much of the existing literature that investigates the dilemma of coming out to a hostile or unwelcoming community has considered the impact of major cultural variables such as race/ethnicity or religion on LGB identity development. Rosario, Schrimshaw, and Hunter (2004) suggested that a long-standing assumption in the literature has been that race/ethnicity is a powerful moderator for LGB identity development, such that those individuals identifying with races/ethnicities that hold traditionally heterosexist values may be at risk for poor or unhealthy identity development and integration. Additionally, the process of integrating one's sexual identity into various social roles such as career or school setting has also been examined (Morris, Waldo, \& Rothblum, 2001). Rostosky 
and Riggle (2002) argued that disclosure of one's LGB identity within these areas was highly dependent on the presence of factors that would ensure the individual's safety (i.e. nondiscrimination policies).

The most recent update of the Guidelines for Psychological Practice With Lesbian, Gay, and Bisexual Clients, the American Psychological Association (APA; 2012) indicated that the contemporary social and political landscapes have witnessed a major revival in the incidence of repathologizing homosexuality. Similarly, Russell and Richards (2003) argued that homophobia, heterosexism, and assumed inequality of sexual minorities are so pervasive in current public discourse that it is impossible to avoid. Given the widespread sociocultural negativity around sexual minorities, individuals who publicly identify as LGB face the threat of discrimination

The extant LGB literature overwhelmingly supports the potential for negative psychosocial health outcomes for LGB individuals exposed to the insidious effects of discrimination (e.g., Meyer, 2007; Meyer, 2013). Sexual minorities may experience discrimination on multiple levels: overtly and covertly, intentional and unintentional, discrete and indiscrete, and in both micro- and macro-level domains (Swim, Johnston, \& Pearson, 2009). Because of this, LGB individuals regularly encounter discrimination in their daily lives, within their normal social and professional functioning. The literature demonstrates that having a well developed, and fully integrated LGB identity decreases the risk of the deleterious effects associated with being a minority (Cass, 1984; Fassinger $\&$ Miller, 1996). Synthesis of one's identity, however, may mean exposure or increased vulnerability to increased levels of discrimination. That is, full disclosure of identity across social contexts may result in individuals becoming more visible or likely targets 
for discriminatory actions. Still not fully understood are the individual factors, such as resilience and stress appraisal that aid LGB-identified people in protecting themselves against toxic environmental influences and events in order to maintain a healthy LGB identity.

\section{Relevance to Counseling Psychology}

The history of mental health treatment for the LGB population, as with many other professional and social institutions, reflects a long tradition of heterosexism (Rothblum, 2000). Beginning in the earliest days of professional psychotherapy, and continuing with some regularity until the 1990 s, it was not uncommon for men with homosexual, or gay, tendencies to undergo reorientation or conversion therapy (Rothblum). This practice was intended to "reorient" the sexual orientation of these clients in order to restore their ability to engage in and enjoy "normal" (or heterosexual) sex practices. By the 1990s, however, a large body of literature had amassed concluding that conversion therapy was not only ineffective, but grounded in unsound methodology, and likely harmful to its recipients (Gonsiorek, 1991). Despite these findings, practitioners continued its use, and heterosexist leanings in the field persisted. The National Association for the Research and Therapy of Homosexuality, for example, was founded by conversion therapists (Rothblum, 2000). Finally, in 2000, Division 44 of the APA issued a statement warning clinicians against the unethical practice of conversion therapy, distancing itself from those in the field who continued to support its utility.

LGB utilization of mental health services has been steadily increasing, beginning in the last decade. Research suggests that as many as $99 \%$ of practicing clinicians have treated at least one client who identified as LGB (Perez, DeBord, \& Bieschke, 2000). 
Despite this, however, gay consumers often reported that their issues were inadequately addressed in treatment by heterosexual clinicians (Cochran, 2001; Perez et al., 2000). Similarly, psychologists report feeling unprepared or poorly trained to serve this population (Perez et al., 2000). Possible explanations for this deficiency range from a dearth of sufficient scientific research aiding mental health professionals to better understand the psychological needs of LGB clients (Cochran, 2001) to clinicians' persistent subtle heterosexist biases (Perez et al., 2000). Unlike many other cultural minorities, the LGB population can and has remained largely invisible; access being granted to researchers only through convenience sampling or at large scale Gay Pride events for which comparison group equivalents do not exist (Cochran, 2001).

Additionally, the tendency to pathologize homosexuality continues to be common in clinical settings where heteronormative biases are strong, and which serve as a barrier for LGB-identified clients seeking treatment (Neville \& Henrickson, 2005). Common practices such as inquiring about marital status or overemphasizing the role that sexual orientation has in perpetuating risky health behaviors all reflect non-normative assumptions about homosexuality. Brown (2005) cautioned clinicians to be aware of this bias; as with all multicultural therapy, interpreting clients' mental health concerns as a result of present minority status may drive them away. That is, assuming the etiology of a mental health concern is linked to one's minority identity may be seen as further discrimination. She stated that "in order to effectively work with [LGB] clients, a therapist must be able to avoid making the client's sexual or gender orientation the problem, and rather focus on the distress that brings the client into treatment" (Brown, 2005, p. 351). 
However, high rates of comorbidity exist between sexual minority status and poor mental and behavioral health outcomes (Barker, 2008). Greater incidence of depression, anxiety, substance use, suicidal behavior, poor sexual health, poor fitness and eating disorders, and other clinical and subclinical concerns are often identified in the LGB population (Barker, 2008; Cochran, 2001). In addition to these, another serious mental health issue exists for the LGB population in the form of internalized homophobia (Fassinger, 2000). The relationship between homosexuality and mental health problems can be explained by the deleterious effect of stress related to experiencing social discrimination as a result of minority status (Cochran, 2001).

Sexual orientation as a cultural identity is a relatively young concept (Broido, 2000), and individual differences vary widely among the members of the LGB population. So much so, in fact, that many have argued against its usefulness as a construct of shared identity (Broido, 2000; Reynolds \& Hanjorgiris, 2000). What is clear, however, is that the experience of being a sexual minority amid an often hostile or rejecting dominant culture is shared and common among LGB individuals. This fact is largely the reason that identifying as a sexual or gender minority is associated with cultural minority status, regardless of any weaknesses inherent to this problematic construct (Broido, 2000).

Given psychology's history of pathologizing homosexuality, and with consideration for the widespread systemic barriers to equitable treatment for sexual minorities, especially in healthcare settings, it is critical that our field continues to take the lead in promoting wellness and mental health for this population (APA, 2012). Counseling psychologists are well equipped for this. Counseling psychology's APA 
Division 17 has historically been on the front line of multiculturalism within the greater discipline of psychology. Division 17 was one of the hosts of the National Multicultural Conference and Summit, held in January of 1999, and more importantly, has traditionally "been influential in addressing racism, sexism, heterosexism, and other forms of prejudice and discrimination" (Sue, Bingham, Porche-Burke, \& Vasquez, 1999, p. 1061). From a multicultural perspective, it is imperative that counseling psychologists continue to be versed in positive approaches to psychology, as with affirmative psychological services for LGB individuals (Riggle, Whitman, Olson, Rostosky, \& Strong, 2008).

The majority of the extant literature has been concerned primarily with the negative experiences associated with the LGB population, such as stress and poor health outcomes (Riggle et al., 2008). In recent years, however, there has been a marked rise in the number of studies conducted to investigate the positive factors that contribute to the successful and healthy development of sexual minorities, such as resilience (e.g., Herrick et al., 2011; Meyer, 2010; Saewyc, 2011; Zimmerman \& Brenner, 2010). This shift is reflective of the field's ongoing mission to better serve this population, through more effective education, training, and science (APA, 2012). It has been widely acknowledged that to date there remains insufficient empirical work concerned with studying resilience and other strength-based factors that may contribute to positive outcomes for sexual minorities (Herrick et al., 2011; Riggle et al., 2008).

\section{Definition of Terms}

For the purposes of the current study it will be necessary to define selected specific terms, fluency with which will be critical for understanding the following work. Of primary importance will be a thorough understanding of terminology specific to 
sexual minority identity theory. Because of the mutable and expanding nature of this area of research, however, it is noted that this will not be a comprehensive effort to define or clarify existing language, but simply serve to highlight how specific terminology is most commonly used within the literature at this time. Given the self-applied nature of many of the following terms, I caution readers not to assume that they can be applied uniformly to every individual who may identify as a sexual minority.

Indeed, a clear definition for the term sexual minority itself has not yet been universally accepted (Savin-Williams, 2001). This is partly a result of the significant within-group differences that exist under the sexual minority rubric (Savin-Williams, 2001; Worthington \& Reynolds, 2009). Another factor is the distinction between those who experience same-sex attractions and those who openly self-identify as gay, lesbian, or bisexual. Savin-Williams defined sexual minorities as individuals who "reject, for personal or political reasons, cultural definitions of any sexual category" (p. 11). Others have taken issue with the term itself, as it connotes oppressed status, potentially complicating the process of self-identification (Balsam \& Mohr, 2007; Reynolds \& Hanjorgiris, 2000).

For the purposes of the current study, however, the term sexual minority will refer to an individual who identifies, publicly or privately, with any sexual orientation that is not strictly heterosexual, which is assumed to be the dominant or majority sexual orientation in the United States. This primarily includes gay men, lesbians, and bisexuals. Also frequently included under this rubric in the literature are transgender individuals. Given the complex and unique issues specific to the development of a gender minority identity, however, this subgroup will not be included in the current 
investigation. This is consistent with recommendations in the current literature (APA, 2012; Brewster 2012). Rather, herein I will be referring to sexual minority and LGB individuals interchangeably, and unless otherwise noted, my assertions will not presume to address the experiences of transgender individuals.

Similarly, sexual orientation is a common term that has evolved in the literature. Traditionally, sexual orientation refers to the biological sex of individuals, to whom one is typically attracted romantically and sexually, including members of one's sex, members of the opposite sex, or both (APA, 2012). This has often been conceptualized as a binary construct (straight versus gay), but which has more recently undergone reevaluation. Past and current research has suggested that sexual orientation exists on a continuum, and in many is fluid, rather than clearly defined boundaries of attraction (APA, 2012).

Arguably integral to sexual minority identity is the coming out process, which refers to "recognizing and accepting that one's primary or predominant sexual orientation is [gay, lesbian, or bisexual] within the context of a heterosexist and homophobic society" (Fassinger \& Miller, 1996, p. 54). Although coming out is considered to be a critical event in the development of one's sexual minority identity, the term often implies public disclosure of identity status (APA, 2012; Fassinger \& Miller, 1996), the problematic nature of which is a critical assumption of the present study. Coming out is contrasted by closeted (i.e., "in the closet"), which refers to LGB individuals who, for reasons of personal safety or privacy, are secretive or cautious about their sexual minority identity, and who publicly disclose their identity to few or no others (APA, 2012). 


\section{Review of the Literature}

\section{Sexual Minority Identity Development}

Following the removal of homosexuality as a diagnosable psychopathology from the third edition of the Diagnostic and Statistical Manual of Mental Illness (DSM-III; 1980), several theorists have attempted to understand the process of sexual orientation identity development (Cass, 1984). Many of these early models focused primarily on the internal processes and conflict resolution related to the adoption of a sexual minority identity (Bilodeau \& Renn, 2005). Little attention was paid in these models to more external social and environmental factors that may be facilitative or disruptive to the identity development process. Characteristic of virtually all of the early models of sexual identity development, however, was the assumption of a stage-based process of change, commonly referred to as the coming out process (Bilodeau \& Renn, 2005). Prior to this notion of a stage-based process, coming out was often conceptualized as a single event wherein the individual publicly disclosed his or her LGB identity.

Among the stage-based models of identity development that emerged during this period was Cass' (1979) six-stage theoretical model of homosexual identity formation. Cass' model drew largely from the extant literature of the time, and integrated commonly theorized stages from various three-, four-, and five-stage models of identity development (Cass, 1984). The six stages that she identified have since been empirically validated by at least two separate studies and have spawned a number of widely documented and accepted measurements (Baker, 2008). Cass' six-stage model is often considered a theoretical benchmark in sexual identity development (Reynolds \& Hanjorgiris, 2000). 
Embedded within each of the six stages of Cass' (1979) model of sexual identity formation are anchors addressing the cognitive, affective, and behavioral functioning typically associated with each stage. The six stages are as follows: (1) Identity Confusion, in which individuals begin to perceive that their behaviors may be defined as "homosexual"; (2) Identity Comparison, in which the potentiality of a homosexual identity has been accepted, and the individuals begin to assess the differences between themselves and heterosexual others; (3) Identity Tolerance, in which the pursuit of meeting same-sex social and emotional needs is seen as necessary rather than desirable; (4) Identity Acceptance, in which increased contact with the homosexual community or culture encourages individuals' own self-acceptance of their identity; (5) Identity Pride, in which pride and loyalty to homosexuals as a group is developed, while increased distance and deidentification with heterosexuals as a group occurs; and (6) Identity Synthesis, in which positive contact with heterosexuals helps to soften the rigidity of the previous stage, and individuals begin to see their sexual identity as only one part of their overall character. In this stage, according to Cass, identity disclosure becomes a "nonissue.” As with other identity development models (e.g., D’Augelli, 1994; Troido, 1988), Cass (1984) acknowledged that no stage has a fixed duration, that regression can occur, and that at any point identity foreclosure may take place, preventing the individual from advancing further.

Cass (1984) argued that one's overall identity or self-image is a composite identity; that is, "a person's theory held about self with regard to social situations, and derives out of interaction with others," (p. 144). According to Cass, composite identities are comprised of individual typological identities, of which sexual identity is one. 
Typological identities are formed as a result of the synthesis of one's self-perception with one's belief about how he or she is perceived by others. One's homosexual identity (heretofore referred to as LGB or sexual minority identity) is an example of a typological identity (Cass). This has become an important distinction, and the interplay between socially derived identities is of principal focus in the present study.

Cass' (1979) model of LGB identity development is central to this area of study. In not only developing her theoretical model, but also subjecting it to rigorous empirical testing, Cass (1984) helped to usher LGB studies away from a medical model of pathological etiology, and towards the conceptualization of interacting internal and external processes that is widely accepted today (Bilodeau \& Renn, 2005). Despite the contribution of Cass' model, however, it deemphasizes the possible deleterious consequences of disclosing one's LGB identity. Cass suggested that ultimately identity disclosure will ideally be a "non-issue" for those who are highly identified; this is largely dependent, however, on the environment in which the disclosure takes place, as well as to whom it is directed. Although Cass' conceptualization of the typological nature of sexual identity is helpful, it may not be sufficiently reflected within the stages themselves. Similarly, the focus on public disclosure of one's identity as a developmental marker implies that lack of disclosure is indicative of arrested development (Fassinger \& Miller, 1996).

In answer to the relative shortcomings of stage models such as Cass' (1979), several theorists began to consider the social context in which identity disclosure occurred. McCarn and Fassinger (1996) developed an inclusive model of sexual minority identity formation, which was later empirically validated (Fassinger \& Miller, 1996). 
Drawing on the work of Allport (1954, as cited by Fassinger \& Miller, 1996), the new perspective of identity formation "assumes the primacy of oppressive environmental influences on normative psychological processes," (p. 54). That is, there is an understanding that LGB identity is not simply a social construction, but also reflective of a historical, political, and socio-cultural context. According to Fassinger and Miller, a critical flaw in the theoretical models developed by Cass and others, is their indiscriminant application across gender and cultures. The implicit assumption of the breadth of those theories is that the experience and process of becoming LGB identified is similar or identical for all individuals, regardless of race or ethnicity, religious background, geographical location, family upbringing, and socioeconomic status.

As with Cass (1979), the model of identity development proposed by McCarn and Fassinger (1996) is structured around distinct stages, or phases as the authors refer to them (to emphasize their more flexible nature). Uniquely, however, the authors conceptualized identity development as occurring across two parallel dimensions: the individual identity development as well as the group membership identity development. Within each of their four stages (Awareness, Exploration, Deepening/Commitment, and Internalization/Synthesis), McCarn and Fassinger identified the developmental processes associated with individual as well as group identity. Additionally, they examined three attitude areas at each phase: attitudes toward self, attitudes toward other LGB identified individuals, and attitudes toward heterosexuals (Fassinger \& Miller).

Importantly, Fassinger and Miller (1996) emphasized that public disclosure of identity status is used only to a limited extent as evidence of development. According to the authors, "disclosure is so profoundly influenced by contextual oppression that to use 
it as an index of identity development directly forces the victim to take responsibility for his or her own victimization" (p. 56). Most relevant to the present study, however, is the understanding that the context of separate individual roles may differ, such that one can comfortably be open about sexual identity among friends, for example, but may choose not to disclose it in a professional setting. As long as the individual has addressed the dilemma, however, he or she is not considered to be any less developmentally integrated according to Fassinger and Miller's model.

As indicated by the literature (see Vaughn \& Waehler, 2010), the stage-based developmental model is still largely accepted within the area of LGB identity. It is important to note that existing models of development are moving away from the focus on public identity disclosure as a developmental benchmark (Fassinger \& Miller, 1996).

However, some degree of identity synthesis or integration is still regarded as the apex of the developmental trajectory, which is a central assumption of the present study. It is suggested that the degree to which one's LGB identity is integrated into his or her overall identity may be extremely dependent on protective resources (i.e. resiliency) and favorable environmental factors (i.e. low perceived discrimination and social support). The daily contexts in which these internal and external forces interact, one's social and professional roles for instance, may also be important.

\section{Identity Integration}

While later theorists have built upon the first LGB identity theory models, the construct of integration continues to be problematic (Legate et al., 2012). Previous research has linked identity integration with positive mental health outcomes (Morris, Waldo, \& Rothblum, 2001). Morris et al. found that psychological distress was inversely 
related to outness for lesbians and bisexual women $(n=2,401)$ in a structural equation model of predictors and outcomes of outness, which was consistent with earlier research with gay men. Kahn (1991) suggested that outness "is associated with integration of personality, psychological health, and authenticity in interpersonal relationships" (p. 47). According to Legate et al. (2012), however, psychological benefits of being out are dependent upon the social context in which disclosure occurs. Using a self-determination theory framework, the authors investigated the variability in levels of autonomy support and control experienced by LGB individuals across social contexts, and how this was associated with the likelihood of identity disclosure. Results indicated that disclosure was more likely to occur in social contexts that were rated to be supportive of autonomy, which was also associated with greater psychological well-being. Conversely, disclosure was significantly less likely to occur in controlling environments, which were associated with lower context-specific self-esteem, as well as greater rates of depression and anger. Clearly, the social and professional contexts in which individuals operate on a daily basis are variously conducive to public disclosure of one's LGB identity.

In the first study of its kind, Waldo (1999) used the minority stress theory to develop models of antecedents and outcomes to heterosexism in the workplace. The theory of minority stress here suggests that LGB individuals experience negative psychological effects as a result of being a minority in nearly every environment in which they exist. Unlike many other cultural minorities who grow up in the context of their cultural identification, however, and who presently may remain surrounded by similarly identified individuals, members of the LGB population are almost always isolated from others like them outside of specific social interactions (Waldo). In the study, Waldo 
hypothesized that the experience of minority stress, when controlling for general jobrelated stress, would be associated with negative psychological, health, and professional outcomes. In addition, Waldo hypothesized that individuals who were less publicly identified (or "out") would experience indirect heterosexism and individuals who were more "out" would experience direct heterosexism. Outness, or the degree to which one publicly identified as LGB, however, was also expected to be higher in work settings where heterosexism was less tolerated at an organizational level (Waldo).

Waldo's (1999) study included two samples $(n=287)$ from distinct geographic locations in the U.S., and represented a $90 \%$ response rate. All participants completed the Workplace Heterosexist Experiences Questionnaire (WHEQ) and the Organizational Tolerance for Heterosexism Inventory (OTHI), both of which were developed for the study. The WHEQ contains 22 items, and was developed to assess employees' experiences of sexual-orientation-based harassment and discrimination by "encompassing experiences ranging from subtle slights...to overtly hostile harassment" (p. 233). The OTHI, also developed for this study, was based on a measure of sexual harassment tolerance. It includes four vignettes followed by three questions, which ask employees to rate the outcomes they believe most likely to occur if an employee complained about heterosexist incidences. Finally, the participants were asked to complete three items designed for this study to estimate outness, or the degree to which they are open about their sexual orientation both in their workplace and their life in general. This is relevant to the present study because it acknowledges variance in outness across different domains of individuals' social functioning. 
The results indicated that LGB individuals who perceived their workplace as being tolerant of heterosexism were more likely to experience it (Waldo, 1999). Conversely, those who perceived their workplace as being intolerant of heterosexism experienced it to a far lesser degree. This suggests important differences between workplace managers' stances on heterosexism: if it is believed to be tolerated in the workplace, it is more likely to be experienced, whereas if it is believed that it is not tolerated it is less likely to be experienced. Waldo also found that outcomes for experienced heterosexism were related to such undesirable sequelae as higher levels of psychological distress, health-related problems, and low job satisfaction.

Waldo's (1999) findings have important implications for the equitable treatment of LGB individuals. Perhaps of even greater consequence, however, is the tacit recognition that social contexts are variable in their acceptance and support of the LGB population. Moreover, it is the LGB individuals' perception of this acceptance and support that is relevant. If heterosexism is perceived on a daily basis, it may have longterm negative effects on the integration of one's LGB identity.

Creed, DeJordy, and Lok (2010) examined the contradiction between individuals' social and professional roles and their LGB identity status. Specifically, the authors examined the experiences of gay ordained ministers, and the institutional contradiction between their expected value system and their LGB identity. The major focus of the study was how "institutionally marginalized people experience and resolve institutional contradictions, and...the implications of this process for their agency" (Creed et al., 2010, p. 1337). The results indicated that experiencing institutional contradictions between one's role in the agency and one's personal identity was often a difficult, prolonged, and 
emotionally-laden process. Positive outcomes were associated with the participants eventually seeing themselves as "change agents" who challenged their institutional marginalization.

Despite Creed et al.'s (2010) findings, the results are not entirely generalizable. Because of the specific nature of the sample (gay-identified ordained ministers), it is difficult to glean from this study how sexual minorities experience institutional contradictions in a broader range of social and professional roles. It is worth noting, however, that the sample was comprised of individuals who already held a leadership role in their community; thus, the process of becoming "change agents" within their specific agency (the church) may have been easier as a result of their status within the agency. In other words, would the reconciliation of institutional values with personal identity be more or less difficult if the agent felt he or she had less power to change the agency? Would these experiences be mirrored in the average parishioner, or would he or she feel more compelled to conform to agency values? In any case, Creed et al. highlight the reality of the difficulties encountered when individuals experience disconnection between their LGB identities and the important roles that they embody.

Perhaps the most notable area in which LGB individuals have experienced institutionally sanctioned discrimination is the United States Military. Historically, the Armed Services maintained a strict exclusionary policy with regard to accepting enlistments from individuals who openly identified as a sexual minority. Disclosure of this identity while enlisted resulted in immediate discharge. Over 32,000 service members were discharged for reasons of sexual orientation between 1980 and 2009 (Burks, 2011). 
In 1993, the Department of Defense adopted a policy, ostensibly for the purposes of ending the prohibition of LGB individuals from enlisting, which became known as “Don't Ask, Don't Tell” (DADT; Burks, 2011). The regulations under DADT allowed sexual minorities to serve but stipulated that matters of sexual orientation were considered personal and private, and were not to be publicly disclosed. Verbal, physical, or sexual behaviors which were considered forms of homosexual conduct were not tolerated and continued to be grounds for discharge. In his review of LGB victimization in the military, Burks (2011) argued that efforts to end overt and systemic discrimination in the military with DADT, likely resulted in continued, albeit more covert, discriminatory policies. Ninety-one percent of LGB respondents of a survey issued by the Office of the Secretary of Defense on sexual orientation and the U.S. military believed that DADT "puts gay servicemembers at risk for blackmail or manipulation" (p. 607). Eighty-six percent and $76 \%$ felt that DADT negatively affected personal and unit relationships, respectively.

Very little empirical, peer-reviewed, research exists with regard to LGB individuals' experience in the Armed Services, but it is known that victimization and discrimination of these servicemembers occurs in the military, whether they are openly identified or not (Burks, 2011). Additionally, according to Burks, the military has long been a setting that cultivates and values conservative gender norms, heterosexism, and sexual stigma. It is estimated that 71,000 to 78,000 sexual minorities currently serve on active duty in the U.S. Armed Services, and at least $37 \%$ had witnessed or experienced some form of harassment or violence in the last year (although given the reluctance of many to disclose their sexual minority identity in this setting, this figure could be 
restricted by underreporting). Between 1994 and 2003 the Servicemembers Legal Defense Network documented an excess of 4,600 incidents of antigay harassment that included verbal and physical abuse, as well as death threats (Burks, 2011). It is important to note that a presidential repeal of DADT was signed on December 22, 2010, and since that time various reports (e.g., Frosch, 2013) have suggested that a climate of increased acceptance of sexual minority servicemembers has been steadily developing. Due to the prolonged and recent nature of the negative impact of DADT on the LGB community, however, it is still relevant for review in the context of the present study.

Given the extremely high numbers of LGB military personnel exposed to harassment and/or discrimination during their service, it is likely that this experience has an impact on their identity development. Additionally, the implicit message behind DADT was for all LGB individuals to "stay in the closet," which similarly does not promote or convey acceptance of LGB identities. Burks (2011) acknowledged that how this message is received or interpreted by LGB servicemembers may vary. Especially relevant to the present study, he also suggested that it may be "moderated by the individual's level of self-acceptance, awareness, or avoidance of LGB identification" (p. 605). This is important because it suggests that the experience of discrimination is moderated by the individual's own identity.

Athletics is another area of social functioning that adheres to a specific set of core values similar to those of the military. Power, dominance, and competition are highly valued in many athletic settings, and driven largely by institutionalized hegemonic masculinity (Anderson, 2002; Jones \& McCarthy, 2010). Qualities typically associated with LGB individuals, specifically gay men, such as gentleness and effeminateness, are 
widely regarded as contradictory to those valued within a hegemonic masculine framework. In other words, "an athlete represents the ideal of what it means to be a man, a definition that contrasts what it means to be feminine and/or gay," (Anderson, 2002, p. 860). Anderson conducted the first qualitative study to examine the experience of being an openly gay male team sport athlete. He conducted a series of interviews with 26 participants who openly identified as gay. Anderson's findings indicated that the athletes experienced the least amount of negativity toward their sexual identity when they successfully contributed to the "overarching motif of sport - winning," (Anderson, 2002, p. 875). Regardless, a framework of heterosexism and homonegativity was uniformly encountered in the form of homophobic dialogue.

Most relevant to the present study, Anderson (2002) found a common theme that he termed "segmented identities" (Anderson, 2002). That is, it was frequently reported that teammates of the gay athletes were aware of the men's sexual identity but did not treat them as gay. This was indicated through such behaviors as asking the men which female classmates they thought were "hot", but never asking which males they were attracted to. This suggests that their teammates were comfortable with having the men on their team as long as behaviors associated with being gay were not explicit (not unlike the DADT culture that has characterized United States armed service). The theme is relevant to the present study because it suggests a kind of covert discrimination or oppression, as though the teammates would not allow the men to fully integrate their identities into their roles as athletes.

The interaction between sexual minority identity and daily, nonculture-bound social and professional roles is important to the present study because it is often in these 
contexts that minority individuals experience the most discrimination (Legate et al., 2012). It is also these contexts in which people often spend the majority of their time.

Given that one's LGB identity status can be "invisible" and only disclosed on a voluntary basis, it is important to understand how the perception of discrimination impacts one's decision to disclose. Sexual minorities are not always in complete control of this decision, however.

In their examination of relationship satisfaction in same-gendered couples, Knoble and Linville (2012) noted that shared outness between partners contributed to greater satisfaction in their relationships. They found that outness served as a "common understanding between partners and as a shared value system," (p. 336) which was important for access to increased social support and greater closeness in relationships. The stress that was associated with outness among couples, though was experienced in part as a result of the fact that degree of desired persona; outness was not always shared between partners, and involvement in a same-gender relationship contributed to increased visibility as a sexual minority. The authors found that participants reported increased family- and work-related stress as a result of shared outness, suggesting that the decision to disclose is not one over which individuals necessarily always have total control.

\section{Perceived Discrimination}

The deleterious effects of minority stress on the physical and mental health outcomes of minority populations are well documented (Meyer, 2013). Accordingly, the minority stress model for explaining disparities in health outcomes has been met with increased interest in health services. Sexual minorities are among the groups that have been identified as at-risk for poor health outcomes. The social stressors that result from 
experiencing commonplace systemic discrimination (at both macro- and micro-levels) have been implicated in the literature as largely responsible for this risk (Meyer, 2007). More specifically, Meyer (2013) outlined a four-stage process by which "distal social attitudes gain psychological importance through cognitive appraisal and become proximal concepts with psychological importance to the individual," (p. 5): (1) the experience of objective stressful events, both chronic and acute, (2) the increase of vigilance secondary to the expectations of these events, (3) the internalization of the perceived negative social attitudes, and (4) concealment of one's sexual orientation as a result of these experiences. To summarize, according to Meyer the effects of experiencing discrimination from one's social environment will accrue to the point at which one becomes protective, rather than open, about one's sexual minority identity status.

It is clear from the literature that discrimination can occur at differing levels of severity and frequency (as in Meyer's [2013] distinction between acute and chronic stress, noted above). According to Sue et al. (2007) in their review of microaggression, it is often the micro-level, routine experience of discriminative experiences that have the most deleterious long-term effects. Swim, Johnson, and Pearson (2009) proposed the importance of considering heterosexist "daily hassles", or "comments or behaviors that reflect or communicate hostile, denigrating, or stigmatizing attitudes and beliefs about [LGB individuals] that are embedded in people's everyday lives" (p. 598). These can be in the form of jokes, expressed stereotypes, insults, exclusion, or other hostile treatment. As opposed to macro-level discrimination, often defined by traumatic but isolated life or historical events, these hassles are more frustrating and irritating everyday occurrences. 
Taken together, however, they represent a distressing and harmful pattern. Daily hassles have been linked to many of the negative health outcomes that result from discrimination outlined above (Swim et al., 2009).

In a longitudinal qualitative study, Swim et al. (2009) attempted to identify common themes in the types of consequences to experiencing heterosexist daily hassles. The predicted themes included: (a) Affective distress, which is characterized by negative mood states and variable attributions of the source of the heterosexism; (b) Self-esteem, which is characterized by increased thoughts of being perceived unfavorably as an individual because of the perception that the LGB community in general was perceived unfavorably; (c) Collective self-esteem, which is characterized by feelings of being an unworthy member of the larger LGB community; and (d) Identification as selfprotection, in which a heightened sense of group-affiliation serves as a buffer against outside threats.

One predicted theme was not supported by their data. Swim et al. (2009) found that those who were more identified experienced more, not less, negative consequences from heterosexist hassles. This finding is concerning, because it seems logical that a stronger identity would be associated with resilience as a protective factor. Similarly, ingroup identification has been shown to be an effective coping mechanism (Swim et al., 2009), so it is notable that these effects were not found to be present. The implications about the protective nature of resiliency of these findings are relevant to the present study.

Eldridge and Johnson (2011) expanded on Swim et al.'s (2009) conceptualization of heterosexism as micro-level discrimination, and proposed broad systemic 
consequences for it, such as social dominance and structural violence. Social dominance refers to "the extent to which one desires that one's in-group dominate and be superior to out-groups" (Pratto et al., 1994, as cited by Eldridge \& Johnson, 2011, p. 388), and structural violence is defined as the "systemic denial of needs for economic well being or for self-determination to certain groups" (p. 386).

Eldridge and Johnson's study included 129 heterosexual adults, ranging from 18 to 70 years of age. All participants, recruited online and by snowball methodology, were asked to complete the following instruments: the Social Dominance Orientation Scale (SDO), the Modern Homonegativity Scale (MHS), the Structural Violence Scale (SVS), and the Attitudes Toward Lesbians and Gay Men Scale (ATLGMS). The SDO is a 16item scale that measures "the extent to which one desires that one's in-group dominate and be superior to out-groups" (Eldridge \& Johnson, 2011, p. 390). The 12 items of the MHS describe "negative attitudes, beliefs, and judgments regarding gay and lesbian people from a systemic as well as individualistic perspective" (p. 390). It is intended to measure the construct of contemporary heterosexism. With its 21 items, the SVS measures attitudes of equality, social justice, oppression, and human rights. The ATLGMS differentiated contemporary heterosexism from "old-fashioned" heterosexism and homonegativism. Previous studies have used it "to measure condemnation-tolerance, hetersexist/heterocentric, and anti-gay attitudes" (p. 391).

The results indicated that social dominance and structural violence are both strongly related to heterosexism. Eldridge and Johnson's (2011) findings are striking in that it is clear that heterosexism as a practice is not exclusively limited to the everyday micro-level discrimination described by Swim et al. (2009), but can also be indicative of 
serious prejudice. The restriction of resources and limiting opportunities for the LGB population is characteristic of significant systemic discrimination, which continues to exist at a macro-level in many contemporary societies.

Both the Swim et al. (2009) and Eldridge and Johnson (2011) studies are important in that they identify the significant risks to psychological well being that LGB individuals are at risk for across a wide spectrum of social functioning. The experience of both micro- and macro-level heterosexism is believed to have negative effects with regard to the integration of one's LGB identity into all social roles. Internal resources such as resiliency, however, may help to serve as protection against the insidious nature of heterosexism that may prevent identity integration from taking place.

\section{Resilience}

Resilience has become an increasingly studied construct in the behavioral and health sciences literature over the last several years (Zautra, Hall, \& Murray, 2010). Despite this, however, definitions of resilience and its purpose have been varied (Smith et al., 2008). Perhaps the most basic definitions of resilience refer to one's ability to "bounce or spring back" (p. 194), but from a stress-perspective, it implies the capacity to effectively return to a basal level of functioning following exposure to a stressor, possibly with little lasting impact (Smith et al.). Most or all definitions of resilience similarly acknowledge the important role of positive adaptability in the face of challenges or barriers that have the potential to result in increased stress and decreased ability to cope (Herrick et al., 2013).

Further consensus on the nature of resilience has not been reached. A debate exists in the literature as to whether resilience is a process or an outcome. Zautra, Hall, and 
Murray (2010), for instance, defined resilience as "an outcome of successful adaptation to adversity" (p. 4, emphasis added). Luthar, Cicchetti, and Becker (2000), however, argued that resilience is a "dynamic process encompassing positive adaptation within the context of a significant adversity" (p. 543). Participating in this debate is beyond the scope of the current study. Rather, I will accept both sides, as I believe they both have their merits and applications. In the interest of defining terminology for this study, I will accept Ungar's (2010) distinction: the term resilient will be used "when referring to positive outcomes despite exposure to risk," and the term resilience will be used when referring to "the individual's participation in processes that lead to well-being under stress" (p. 405).

Over the course of the previous decade, resilience has been increasingly investigated in the literature, with much of the focus on the role of resilience in LGB identity development in the adolescent cohort (Anderson, 1998; Murdock \& Bolch, 2005; Saewyc, 2011; Scourfield, Roen, \& McDermott, 2008). This is understandable, however, as adolescence is the time when emerging sexual minorities are most at risk for negative outcomes. Despite this attention, though, Herrick et al. (2011) suggested that, as an area of study for sub-cultural phenomena, resilience has yet to receive much focus.

Ungar (2010) defined resilience as "the acquisition of both internal and external assets that work together to potentiate a state of mental and physical well-being when individuals are exposed to non-normative levels of psychosocial stress" (p. 405). The preponderance of the $\mathrm{LGB} /$ resilience research to date, however, emphasizes almost exclusively the role that environmental factors (i.e., social support, exposure to positive events) play in fostering resilience in sexual minority individuals. The internal 
predispositions or characteristics which may also lead to resilience are often overlooked. In their examination of stress appraisal and resilience, Lazarus and Folkman (1984), for instance, suggested that as a result of possessing innate strengths, such as a high sense of mastery, may lead resilient individuals to regard stressors as less threatening than nonresilient individuals. Therefore, resiliency serves to protect individuals against stress even before it occurs because it is appraised differently (Meyer, 2010). This idea is particularly relevant to the present study: if resilient LGB individuals are able to appraise discriminatory events or homonegative environments as being less threatening than their non-resilient peers, they may be more successful at maintaining a healthy LGB identity.

Despite the lack of a unifying theoretical model of resilience as a component of LGB identity development, several models exist which may be useful in understanding the role that it may play. Zimmerman and Brenner (2010), for instance, adapted their protective model of resilience from Garmezy, Masten, and Taylor's (1984; as cited in Zimmerman \& Brenner, 2010) work in developmental stressors. This model posits that specific protective factors buffer against harm and negative outcomes in the face of risk. According to Zimmerman and Brenner, these protective factors do not reduce risk of harm itself, but moderate its effect on outcomes, with "the likelihood of a negative outcome [remaining constant] as risk increases in the presence of a promotive factor" ( $p$. 286). This conceptualization is useful to the present study, as it is assumed that discrimination and other negative experiences associated with sexual minority status are not directly reducible or controllable by the individual, only tolerated more or less successfully.

Because of the culture-wide nature of homophobia and heterosexism, Herrick et 
al. (2011) suggested that children are exposed to homophobic messages, and therefore "syndemic processes among [gay men] likely begin at a young age," (Herrick et al., 2011, p. 26). The Centers for Disease Control and Prevention (CDC) defines a syndemic process as "two or more afflictions, interacting synergistically, contributing to excess burden of disease in a population," (Herrick et al., p. 26). In this case, the syndemic process refers to the often co-occurring psychosocial health problems experienced by the LGB population, detailed above.

Herrick et al. (2011) argued, however, that gay men are a resilient population, as evidenced by examples of considerable strength in both the health and historical literature, and in the face of sometimes extreme discrimination. The authors cited health statistics supporting this assertion, and indicated that most significantly it has been the gay community's ability to persevere following the AIDS epidemic that suggests their capacity for resilience. Although Herrick et al.'s claim is empowering to LGB individuals, there has yet been no empirical study to support it (Herrick et al., 2013).

Similarly, in her review of the literature on sexual orientation development in adolescence, Saewyc (2011) suggested that despite significant stress associated with this turbulent period, most LGB adolescents do not develop significant long-term health concerns or pathologies. Rather, the vast majority go on to lead healthy and fulfilling adult lives. She suggested that the same factors that contribute to resiliency and positive outcomes in other adolescents (connectedness to family, peer support, school, other adults or mentors, and religion) also help to buffer sexual minority adolescents from experiencing unhealthy development. These youth are at a disadvantage from their nonLGB peers, however, in that the research demonstrates that LGB adolescents routinely 
have decreased access to these supports (Saewyc). In spite of this, Saewyc maintained, LGB adolescents typically experience healthy developmental outcomes, possibly suggesting that they access protective factors through other channels.

Russell and Richards (2003) investigated resiliency in the LGB community as a protective factor against what they claimed to be a pervasive anti-gay political climate in the United States. According to the authors, heterosexist messages are transmitted via political campaigns, as well as everyday political discourse. In this way antigay politics draw on cultural homonegativity, but contribute to it as well. Among the stressors that are experienced by LGB individuals during political discourse of this nature are distortions and misinformation communicated to the non-gay community about the LGB community, threatening connotations and a sense of danger, stereotyping, and the general assumption from the non-gay community that they are different or "other" (Russell \& Richards, 2003). The authors surveyed a large number $(n=663)$ of LGB individuals after the passage of an antigay legislative amendment in Colorado in order to understand not only the negative consequences of the amendment, but also the more positive consequences indicative of cultural resiliency.

Russell and Richards (2003) indicated five outcomes that were identified as sources of resiliency for the LGB individuals who participated. First was "movement perspective". This was the ability of LGB individuals to cognitively shift from seeing the significance of an isolated antigay event, to viewing it in the context of the greater movement for LGB equal rights. The ability to adopt a broad perspective helps to decrease feelings of isolation and powerlessness, and increase a sense of community mobilization. Second was "confronting internalized homophobia". The authors stated 
that a number of observers have noted that successful antigay politics often paradoxically results in growth within the LGB community, as members seek to distance themselves from their oppressors and so more readily resolve their internalized feelings of homophobia. Third was "expression of affect". Anger and sadness are appropriate emotions in the face of violation and loss, and the expression of these emotions may result in individual motivation to act, which may indicate effective coping. Fourth was "successful witnessing," which is the ability on the part of LGB individuals to seek social support from outside of the LGB community. Last was the "availability of the LGB community" in general, and the ability of LGB individuals to access its resources for information, support, and an overall sense of efficacy.

Russell and Richards' (2003) findings are promising empirical evidence for the importance of resilience as a protective factor for LGB individuals. The findings are also especially relevant to the present study, as they are suggestive of the need for resilience in facilitating the development of a stronger, more integrated LGB identity, despite experiencing discrimination. Not addressed by Russell and Richards' study, however, is the influence that individual social roles have on one's capability for resilience. The authors considered LGB individuals in the context of existing within a greater LGB community, which is an understandable source of strength and support, but does not necessarily consider the effects of antigay politics on those LGB individuals who, for whatever reason, are disconnected from this community.

\section{Purpose Statement}

My purpose for the present study was to better understand the occurrence of LGB identity integration across social roles as predicted by the protective factors of resilience. 
It was hypothesized that resilience (conceptualized both as access to environmental support and personal characteristics and predispositions) as well as less severe and frequent perceived discrimination would predict the degree to which an individual's sexual minority identity was integrated across domains of social functioning. It has been well understood in the literature for some time now that public disclosure of one's identity is an inadequate and unrealistic developmental marker, given the risk of discrimination or rejection often associated with coming out. Despite this, however, it was thought that outness would be associated with more positive developmental characteristics, and less negative characteristics such as internalized homonegativity. Additionally, those who experience discriminatory events, including broad environmental heterosexism, as relatively unthreatening were expected to be highly resilient. In order to draw conclusions about the facilitative effects of resilience of sexual minority identity, the purpose of this study was to (a) determine whether resilience and perception of discrimination were predictive of identity integration, and (b) evaluate the relationship between integration and dimensions of LGB identity development (discussed in detail in the following chapter).

\section{Rationale}

The present study is primarily relevant to the existing literature in three ways. First, it is of clinical interest to better understand the implications of outness on adjustment and mental health outcomes, which continue to be underrepresented in the literature. Although well-developed sexual minority identity status has previously been associated with indicators of positive outcomes and overall good psychological wellness (Morris et al., 2001), the effect of outness on mental health is an area of the literature that 
is infrequently investigated, and for which results have been mixed (Feldman, 2012).

From a theoretical perspective, given the fluid nature of many of the constructs addressed in this study, it is critical that this area of research should be ongoing. Sophie (1986) found that change in sexual orientation was extremely sensitive to social and historical contextual factors, and argued that assessments of sexual minority identity development must acknowledge the influence of these conditions. With many recent and notable developments with regard to policy change (e.g. the variable status of same-sex marriage legislature across state lines), as well as both pro- and anti-gay messages proliferating the current sociocultural rhetoric, it was important that research of this nature remain current and advanced.

Finally, resilience has been conceptualized as both personal characteristics and external support factors (or some combination of the two). With regard to the latter, Meyer's $(2007 ; 2013)$ minority stress model suggests that environmental stressors experienced by minority groups increase the likelihood of poor health outcomes among those groups. Research addressing the stressors, such as discrimination, experienced by minority groups is important for the ongoing development of supportive systemic changes to reduce this threat (see, for example, Waldo [1999], discussed in detail above). As Waldo (1999) argued, it is possible that clearer understanding of the ways minority stress is experienced across social settings can contribute to improvements in accommodations within a majority context.

\section{Research Questions}

The purpose of the present study was to determine the extent to which perceived discrimination, moderated by resilience, predicted sexual minority identity integration. 
Of additional interest are the relationships between perceived discrimination and resilience to various dimensions of LGB identity development.

\section{Research Question One:}

Does resilience (as measured by The Courage to Challenge Scale; Smith \& Gray, 2009) moderate perceived discrimination across areas of social functioning (as measured by the Gay and Lesbian Oppressive Situations Inventory - Frequency and Effect; Highlen, Bean, \& Sampson, 2000) to predict integration of LGB identity, or outness (as measured by the The Outness Inventory; Mohr \& Fassinger, 2000)?

\section{Research Question Two:}

What is the relationship between both resilience and perceived discrimination, and the eight individual theoretical dimensions of LGB identity development (as

measured by the Lesbian, Gay, and Bisexual Identity Scale; Mohr \& Kendra, 2011)? 


\section{CHAPTER 2: RESEARCH METHODOLOGY}

This chapter describes the research design, methodology, and procedures for the present study. The methodology includes: (a) the design, (b) participants, (c) measures, and (d) procedure, including data collection and analysis. The purpose of the study was to determine the extent to which resilience moderates the effect and frequency of perceived discrimination in predicting sexual minority identity integration. Of additional interest were the relationships between perceived discrimination and resilience to the eight individual dimensions of LGB identity development.

\section{Research Design}

In order to establish the relationship between resilience to perceived discrimination and LGB identity integration, a non-experimental, survey-based design was used. It was hypothesized that a range of environmental factors, including frequency and effect of experiencing discrimination, would predict level of identity integration (see Figure 1). Those who were found to report lower frequency of discriminative events, as well as less severe negative effects of such events, were expected to be more highly integrated than those who reported higher frequency of, and more severe effects associated with discrimination. Resilience was proposed to serve as a moderator variable for the sexual minority identity development process, such that those respondents who were assessed to be highly resilient were expected to report less severe effects of discrimination, regardless of frequency. Because resilience is conceptualized as an internal process as well as external, it was expected to moderate the subjective effect of the experience of discrimination more significantly than perceived frequency of 
discriminative events themselves, therefore buffering the negative effects of discrimination in predicting identity integration (see Figure 2).

The study was grounded in McCarn and Fassinger's (1996) theoretical model of sexual minority identity development. Within this model, the highest level of identity development occurs when full commitment and acceptance of one's sexual minority identity has taken place, regardless of whether one publicly discloses this identity or not. It was assumed that it may not be possible to disclose one's sexual minority identity in any or all domains of social functioning for reasons of safety to the individual. McCarn and Fassinger termed this final stage of identity development as "Synthesis/Internalization" to reflect the distinction between integrating or synthesizing one's sexual minority identity with overall identity (in that it is disclosed or otherwise communicated publicly and openly), and internally accepting one's identity while electing to protect it from public recognition. Subsequent literature (Mohr \& Fassinger, 2000; Mohr \& Kendra, 2011) has established that this process can vary across social contexts such that one can publicly identify as a sexual minority in one domain, while continuing to keep his or her identity private in others. The individual factors that are related to this process, specifically resilience to perceived discrimination, are of primary interest to the proposed study.

\section{Participants}

Participants included self-identified lesbian $(n=92,36.8 \%)$, gay $(n=106$, $42.4 \%)$, and bisexual $(n=52,20.8 \%)$ adults. Mean age of participants was approximately 34 , median age was approximately 42 . Of the 250 responses included in the analysis, 138 identified as female (55.2\%), 105 as male (42\%), three as transgender 
$(1.2 \%)$, and four as "other" (1.6\%). Please refer to Table 1 for detailed summary of participant demographics.

The minimum age required for participation was 18 . The reasons for this were two-fold: (1) because the parameters of the study included only those individuals who had a fully developed LGB identity, the formative nature of the teenage years in terms of personal identity development was thought to present a potential confound, and (2) the ethical and logistical considerations for recruiting minors were considered too significant a barrier for the purposes of the study. No upper-limit age restrictions were placed on the responding participants. The varying nature and context of social roles associated with different age cohorts were considered of interest. As compensation for participating, respondents were entered into a drawing to win a $\$ 100$ gift certificate redeemable at the online marketplace Amazon.com.

Preliminary analyses estimated that a suggested sample size of $n \geq 119$ would be necessary to achieve sufficient statistical power for the present study, using one criterion variable and three predictor variables. All power estimates were made using G*Power 3.1 for Mac OS 10.4 (Faul, Erdfelder, Lang, \& Buchner, 2009). These estimates were satisfied by the obtained sample.

\section{Measures}

The web-based questionnaire contained four instruments to investigate each of the constructs, including: (a) identity integration (The Outness Inventory; Fassinger \& Mohr, 2000), (b) resilience (The Courage to Challenge Scale; Smith \& Gray, 2009), (c) perceived discrimination (Gay and Lesbian Oppressive Situations Inventory - Frequency and Effect; Highlen, Bean, \& Sampson, 2000) and (d) LGB identity development 
(Lesbian, Gay, and Bisexual Identity Scale; Mohr \& Kendra, 2011). Permission for use of all instruments, including minor revisions detailed below, was granted by all respective primary authors via personal communication.

\section{The Outness Inventory (OI; Fassinger \& Mohr, 2000).}

The OI was developed as a measure of the degree to which participants' sexual orientation was publicly known and openly discussed with people in the contexts of their social functioning (Mohr \& Fassinger, 2000). An important assumption of the development of this scale was that disclosure of one's sexual identity is not limited to verbal disclosures alone, but rather can occur by a range of both covert and overt nonverbal communications. Therefore, the resultant scale was designed to be sensitive to all levels of communication of sexual identity. A 7-point, Likert-type rating scale was developed which included the following possible responses: $1=$ person definitely does not know your sexual orientation status; 2 = person might know your sexual orientation status, but it is never talked about; 3 = person probably knows your sexual orientation status, but it is never talked about; 4 = person probably knows your sexual orientation status, but it is rarely talked about; 5 = person definitely knows your sexual orientation status, but it is rarely talked about; 6 = person definitely knows your sexual orientation status, and it is sometimes talked about; and $7=$ person definitely knows about your sexual orientation status, and it is openly talked about. A factor analysis resulted in retention of ten of 11 possible items, contained within three subscales: Out to World (including new straight friends, old straight friends, work peers, and work supervisors),

Out to Family (including mother, father, siblings, and extended family), and Out to Religion (including members of the religious community, and leaders of the religious 
community). Though bisexual individuals were not included in the development of this measure, the generalized language choice ("sexual orientation" as opposed to "gay or lesbian identity") in the individual items suggest that it is acceptable for use in the present study.

Validity analyses indicated that the three subscales of the OI conformed to validity measures as expected (Mohr \& Fassinger, 2000). Validity measures included measures of self-esteem, same group orientation ( $\mathrm{SGO})$, other group orientation (OGO), the deepening/commitment $(\mathrm{D} / \mathrm{C})$ and internalization/synthesis $(\mathrm{I} / \mathrm{S})$ phases of sexual identity development (McCarn \& Fassinger, 1996), years since identity milestones, and pro-gay religious involvement. Correlations are reported first for lesbian respondents and second for gay male respondents. Out to Family was positively related to self-esteem ( $r$ $=.14, .10), \mathrm{SGO}(r=.21, .20), \mathrm{OGO}(r=.04, .22), \mathrm{I} / \mathrm{S}(r=.20, .21)$, years since developmental milestones $(r=.18, .03)$, and religion $(r=.21, .02)$. Out to World was also positively related to self-esteem $(r=.15, .21)$, SGO $(r=.31, .31)$, OGO $(r=.15$, $.28), \mathrm{I} / \mathrm{S}(r=.21, .24)$, years since developmental milestones $(r=.08, .08)$, and religion $(r$ $=.25, .08)$. Finally, Out to Religion was positively related to self-esteem $(r=.11, .18)$, SGO $(r=.35, .37)$, OGO $(r=.09, .37), \mathrm{I} / \mathrm{S}(r=.16, .26)$, years since developmental milestones $(r=.19, .29)$, and religion $(r=.59,40)$. Because of the continuing identity development associated with the $\mathrm{D} / \mathrm{C}$ phase, this was the only measure negatively related to all three subscales Out to Family $(r=-.19,-.12)$; Out to World $(r=-.20,-.14)$; and Out to Religion $(r=-.17,-.04)]$.

Cronbach's alpha coefficients were moderate to strong for the full sample across all three subscales: Out to World (4 items; $\alpha=.79$ ), Out to Family (4 items, $\alpha=.74$ ), and 
Out to Religion ( 2 items; $\alpha=.97$ ), suggesting sufficient internal consistency. Although few of the validity correlations found were notably strong, the items performed as predicted. Given these findings and the sufficient reliability, and because a thorough review of the literature did not produce an equivalent or comparable measure as suited for use in the present study, the OI has been determined to be the most appropriate way to assess this variable.

\section{The Courage to Challenge Scale (CCS; Smith \& Gray, 2009).}

Resilience has been subject to increased attention in the social sciences literature over the previous decade, and has been conceptualized in a variety of ways (Smith et al., 2008). One thread of consistency woven through the various definitions suggested has been a focus on the capacity for successful coping. Smith and Gray (2009) suggested three factors that contribute to individual resiliency development: (a) the buffering or mediating effects of a supportive community environment, (b) protective interpersonal relationships, and (c) intrapersonal attributes which enable individuals to "shake off setbacks more easily, to persevere or continue to try when success seems uncertain, and to sustain faith...when others experiencing similar stressors would likely succumb to defeat," (p. 75). The authors argued that the first two factors are domains in which LGB individuals are most likely to encounter discrimination, and so they must rely more heavily on the third factor, defined by the authors as hardiness. This includes the ability to dispute negative social messages regarding one's sexual orientation (e.g., "When I encounter people's hostile attitudes, I can control my reactions.")

The 18 items included in the CCS (Smith \& Gray, 2009) are presented as a 7point, Likert-type rating scale: 1 = strongly disagree; 2 =disagree; 3 = mildly disagree; 4 
$=$ neutral $; 5=$ mildly agree $; 6=$ agree $; 7=$ strongly agree. Validity analyses for the 18 CCS items were completed by examining the correlations between scores on the CCS and scores on measures of convergent and divergent constructs. Because no equivalent instrument measuring LGBT resiliency existed at the time of development, the authors used a measure of gay and lesbian self-esteem to establish convergent validity. It was found that these two measures were positively and significantly correlated $(r=.60, p<$ $.01)$. Additionally, the CCS was found to be negatively and significantly correlated to a measure of general anxiety, a construct presumed to have little similarity to resilience or hardiness $(r=-.09, p<.01)$. Cronbach's alpha coefficient of internal consistency indicated that the scale was reliable across the different sub-groups (lesbians, gay males, bisexuals, and transgender individuals) and geographic locations of three different samples $(\alpha=.86)$. Although this is a brief and simple measure, it remains the only one of its kind, and is therefore of importance to the present study.

\section{Gay and Lesbian Oppressive Situations Inventory - Frequency and Effect}

\section{(GALOSI-F \& -E; Highlen, Bean, \& Sampson, 2000).}

The GALOSI consists of situations characterized by hetereosexism that sexual minorities may commonly encounter (e.g., "I have had anti-LGB remarks directed at me.”). Each situation is rated on two different 5-point, Likert-type scales for both frequency (49 items) and effect (47 items). The frequency scale ranges from 1 (Never) to 5 (Almost Always), and the effect scale ranges from 1 (No Effect) to 5 (Extremely Strong Effect). Factor analysis determined the presence of seven subscales, which Highlen et al. (2000) identified as Couples Issues (CI); Danger to Safety (DS); Exclusion, Rejection, and Separation (ERS); Internalized Homonegativity (IH); Restricted Opportunities and 
Rights (ROR); Stigmatizing and Stereotyping (SS); and Verbal Harassment and Intimidation (VHI).

Cronbach's alpha coefficients of internal consistency of the GALOSI-F scales were determined to be moderate to strong: CI ( 4 items; $\alpha=.63$ ); DS ( 5 items; $\alpha=.77$ ); ERS ( 9 items; $\alpha=.87)$ ) IH (10 items; $\alpha=.88)$; ROR (3 items; $\alpha=.69$ ); SS (11 items; $\alpha=$ $.85)$; and VHI (7 items; $\alpha=.77$ ). Correlations between the GALSOI-F subscales and the Impression Management scale (a measure of desirable responding) were reported as estimates of discriminant validity: CI $(r=-.02)$, DS $(r=.02)$, ERS $(r=.01)$, IH $(r=-.10)$, ROR $(r=-.08)$, SS $(r=-.03)$, VHI $(r=-.15)$. The low and nonsignificant correlations were interpreted as an indicator that there was no association between responses to the GALOSI-F items and socially desirable responding in general.

Cronbach's alpha coefficients of internal consistency of the GALOSI-E scales were determined to be moderate to strong: CI ( 3 items; $\alpha=.77$ ); DS ( 6 items; $\alpha=.89$ ); ERS ( 10 items; $\alpha=.93)$; IH ( 9 items; $\alpha=.91)$; ROR ( 3 items; $\alpha=.78)$; SS ( 8 items; $\alpha=$ .88 ); and VHI ( 8 items; $\alpha=.84$ ). Correlations between the GALSOI-F subscales and the Impression Management scale (a measure of desirable responding) were reported as estimates of discriminant validity: CI $(r=-.09)$, DS $(r=.03)$, ERS $(r=.08)$, IH $(r=-.02)$, ROR $(r=-.10)$, SS $(r=.01)$, and VHI $(r=-.13)$. The low and nonsignificant correlations were interpreted as an indicator that there was no association between responses to the GALOSI-F items and socially desirable responding in general.

\section{Lesbian, Gay, and Bisexual Identity Scale (LGBIS; Mohr \& Kendra, 2011)}

Forty-one items were included in the exploratory factor analysis of the LGBIS (Mohr \& Kendra, 2011). A total of 25 items were minimally reworded from the original 
Lesbian and Gay Identity Scale (LGIS; Mohr \& Fassinger, 2000) to reference sexual orientation as $L G B$ as opposed to lesbian or gay. For all items, participants were asked to rate their experience as a LGB individual using a 6-point Likert-type rating scale $(1=$ disagree strongly; 2 = disagree $; 3=$ disagree somewhat $; 4=$ agree somewhat $; 5=$ agree $;$ 6 agree strongly). Following all factor analyses, a total of 27 items were retained for the final version of the LGBIS, with a total of eight revised orthogonal subscales: Acceptance Concerns (three items; e.g., "I often wonder if others judge me for my sexual orientation.") refers to the degree to which one is concerned about whether one's identity will be negatively perceived by those around them; Concealment Motivation (three items; e.g., "I prefer to keep my same-sex romantic relationships rather private.") refers to the extent to which one is motivated to keep one's sexual minority identity private; Identity Uncertainty (four items; e.g., "I'm not totally sure what my sexual orientation is.") is the degree to which one is uncertain about whether one is homosexual, bisexual, or heterosexual; Internalized Homonegativity (three items; e.g., "If it were possible, I would choose to be straight.") refers to the negative feelings and beliefs one holds about one's own sexual minority identity; Difficult Process (three items; e.g., "Admitting to myself that I'm an LGB person has been a very painful process.") refers to the level of personal difficulty one experiences during the process of LGB identity development; Identity Superiority (three items; e.g., "I look down on heterosexuals.") measures the strength of one's belief that sexual minorities are superior to heterosexuals; Identity Affirmation (three items; e.g., "I am glad to be an LGB person.") measures the positive feelings (e.g., pride) one has about being a member of the LGB community; and Identity Centrality (five items; e.g., "Being an LGB person is a very important aspect of my life.") measures 
the degree to which one's sexual minority identity is central to one's overall identity. The final subscale was of critical importance to the present study for the purposes of confirmatory analyses discussed in the following chapter.

Validity analyses of the LGBIS indicated that all subscales conformed to validity measures as expected (Mohr \& Kendra, 2011). Correlation results from exploratory and confirmatory factor analyses were both reported, in that order. Acceptance Concerns were negatively associated with measures of public collective self-esteem $(r=-.20,-.33)$ and outness $(r=-.55,-.58)$, and positively associated with a measure of ego-dystonic homosexuality $(r=.41, .45)$. Concealment Motivation was negatively associated with outness $(r=-.55,-.58)$, and positively associated with ego-dystonic homosexuality $(r=$ $.34, .43)$ and a measure of self-concealment $(r=.40, .51)$. Identity Uncertainty was negatively associated with the number of years since reaching LGB developmental milestones $(r=-.27,-.24)$. Internalized Homonegativity was positively associated with ego-dystonic homosexuality $(r=.83, .85)$ and negatively associated with the degree of interest in interacting with other homosexuals, reported as SGO $(r=-.41,-.43)$. Difficult Process was positively associated with ego-dystonic homosexuality $(r=.47, .51)$. Identity Superiority was negatively associated with the degree of interest in interacting with heterosexuals, reported OGO $(r=-.38,-.47)$. Identity Centrality was positively associated with a measure of identity importance $(r=.76, .81)$ and SGO $(r=.55, .53)$. Identity Affirmation was negatively associated with ego-dystonic homosexuality $(r=$ $.56,-.64)$ and positively associated with identity importance $(r=.41, .44)$ and SGO $(r=$ $.73, .73)$. All relationships were found to be significant $(p<.05)$. 
Test-retest reliability of the LGBIS was determined to range from sufficient to excellent after a 6-week period (Mohr \& Kendra, 2011). Cronbach's alpha coefficients of internal consistency for Time 1 and Time 2 are both reported, in that order, as follows: Acceptance Concerns $(\alpha=.82, .74)$; Concealment Motivation $(\alpha=.72, .72)$; Identity Uncertainty ( $\alpha=.93, .89)$; Internalized Homonegativity $(\alpha=.89, .93)$; Difficult Process $(\alpha=.86, .88)$; Identity Superiority $(\alpha=.82, .83)$; Identity Affirmation $(\alpha=.94, .91)$; and Identity Centrality $(\alpha=.84, .83)$. The LGBIS was designed for individual interpretation of each subscale to capture the multidimensional nature of LGB identity; therefore, an estimated Cronbach's alpha for the overall survey was either not generated or reported.

\section{Demographics}

Respondents were asked to complete general demographic items including age, race/ethnicity, gender/sex, sexual orientation, religious orientation, relationship status, occupation, education, and state of residence. Response options for the sexual orientation included transgender along with lesbian, gay male, and bisexual, although it was not expected that enough transgender individuals would be found by sampling procedures to garner sufficient statistical power to consider this group.

\section{Procedure}

\section{Survey}

All four instruments described above were presented to the participants in a sequence randomly generated by the online portal hosting the survey (Qualtrics). Each instrument was presented in full. In addition to the demographics questionnaire (see Appendix A), the specific content of the survey itself is summarized as follows: (1) the 10 items of the OI (Mohr \& Fassinger, 2000; see Appendix B); (2) the 18 items that 
comprise the CCS (Smith \& Gray, 2009; see Appendix C); (3) The GALOSI-F and GALOSI-E (Highlen et al., 2000; see Appendix D) for a total of 55 items; and (4) the 27 items of the LGBIS (Mohr \& Kendra, 2011; see Appendix E). All items were presented in the sequence recommended by their respective authors.

The only instrument selected for use in the proposed study that required minor revisions to individual item wording was the GALOSI-F and -E (Highlen et al., 2000). Most of the 55 items on this scale refer to sexual orientation as gayness (e.g., "It has been hard for me to accept my gayness") and sexual minority identity as gay (e.g., "I have known gay people who have attempted suicide"). In order to be more inclusive to the intended sample, we chose to reword such items to reflect a broader range of sexual orientation and identity (e.g., "It has been hard for me to accept my sexual orientation," or "I have known LGB identified people who have attempted suicide"). A few items were written to refer specifically to gay men (e.g., "I have seen people assume that gay men are HIV positive") and will remain unchanged. It was thought that statements such as these still reflected a level of discrimination towards the sexual minority community as a whole that their specific wording would not significantly alter how participants responded.

The decision to impose minor language revisions to a scale in order to reflect broader inclusion of sexual orientation parameters is not unprecedented in the literature. During the development of the LGBIS (Mohr \& Kendra, 2011), the authors made similar changes to several items on the original LGIS questionnaire. The intention of Mohr and Kendra, as ours was here, was to reduce the specificity of the language choices in the LGIS (using the words lesbian or gay only) by referring more broadly to sexual 
orientation. As with the original, more restricted version of this measure, the authors obtained acceptable and comparable estimates of reliability and validity. This suggests that appropriate items can be reasonably reworded in order to reflect a more inclusive interpretation of sexual orientation, and the strength and clarity of the construct reflected in the overall instrument will not suffer.

\section{Data Collection}

Data for this study were collected nationally, with 38 U.S. states represented in the total sample, between October 2013 and February 2014. Recruitment occurred across two separate phases. Initially, internal review board approval was sought and granted allowing for dissemination of the online survey to occur exclusively via direct email communication with LGB community center sites and agencies, located through public internet directories. When attempts to garner a sufficiently large sample through previously-approved routes failed, an amendment to the original IRB proposal was filed and subsequently approved. This amendment allowed recruitment efforts to expand to the greater online community, including use public and private APA and university listservs, individual community centers, online message boards, and social networking sites utilized by LGB organizations (e.g. Facebook). See Appendices F-H for information regarding internal review board approval and informed consent for the present study.

A total of 548 people responded to the online survey request. Of these total overall responses, 279 were determined to be incomplete and were not included in the final analysis. An additional 19 responses were excluded due to sexual orientation selfreported as other than lesbian, gay, or bisexual, or failing to disclose this information. 
Ultimately, 250 responses were included in the final analysis, representing a usable sample of $46 \%$ of total responses obtained.

\section{Statistical Analysis}

Alpha levels for all tests were set to .05 . Prior to completion of any statistical analyses an analysis of missing values was completed. According to results of this analysis, $37.2 \%(n=93)$ of all individual cases contained some degree of missing values among their responses. Further analysis, however, revealed that of all 41,500 single response values collected, only $2.7 \%(n=1121)$ were missing or incomplete.

Some have suggested that missing values under 5\% may be ignored with little detriment to accuracy of findings (Field, 2009), but the given observed non-random distribution of missing values among these data, it was decided that replacing missing values was necessary. Use of multiple imputation for the purposes of replacing missing values was initially considered but ultimately disregarded for two reasons: (1) the slight but notable non-random distribution in missing values would have violated a core assumption of imputation, and (2) following an experimental run of this test, it was determined that results of subsequent statistical analyses (e.g., hierarchical regression, described below) using imputed data did not differ significantly from results using data with replaced series' means. Because use of series means was a less elaborate and in this case equally effective method for replacing missing values, it was decided upon as an appropriate and sufficient method for accounting for missing data

To investigate predictors and moderators of identity integration, a hierarchical multiple regression statistical analysis was utilized. According to Tabachnik and Fidell (2006), multiple regression is an appropriate statistical analysis for the main research 
question in that multiple possible predictors of identity integration were observed, as well as the degree to which these variables were predictive. Additionally, Frazier, Tix, and Barron (2004) indicated that multiple regression is similarly appropriate for establishing moderating effects of continuous variables. For the present regression model, the dependent variable (DV) is identity integration, or outness, as measured by the OI (Fassinger \& Mohr, 2000). The continuous independent variables (IVs) were frequency and effect of perceived discrimination, as measured by the GALOSI-F and -E (Highlen, Bean, \& Sampson, 2000). Resilience, as measured by the CCS (Smith \& Gray, 2009), was entered as a moderator variable.

In keeping with Frazier et al.'s (2004) comprehensive guidelines for testing moderator effects in counseling psychology research, the following steps were completed prior to structuring the regression equation: First, all continuous data were standardized using $z$ scores. The authors suggested that this process was important for reduction of problems with multicollinearity as well as to improve ability to plot significant moderator effects. This is also preferred to using artificial cut-offs (e.g. median splits) because it retains the continuous nature of the variables, which can result in a loss of information, as well as a reduction of power. Second, product terms were created in order to represent the interaction between the proposed predictors (effect and frequency of perceived discrimination) and moderator (resilience). Because the purpose of this study was to investigate the moderating effects of resilience on effect and frequency of perceived discrimination, only the interaction effects between those variables were included in the regression model as product terms (Frazier, personal communication, 2014). 
Hierarchical regression equations are typically structured with priority given to the most theoretically or scientifically supported variable over additional variables (Field, 2009). Despite the amount of available literature considering discriminative experiences as predictive of negative outcomes in minority groups (see Meyer, 2007) resilience was given priority in the proposed regression equation. The rationale for this decision is supported by Tabachnik and Fidell (2006), who argued that point of entry for IVs may also be determined by including lesser known variables first, followed by more robustly studied variables, such that "the major set is evaluated for what it adds to the prediction over and above the lesser set" (p. 166). Because resilience is the lesser-studied variable among this population, it was given first point of entry in the regression equation.

Subsequent points of IV entry were given to effect and frequency of perceived discrimination, in that order. As noted, the GALOSI (Highlen et al., 2000) yields two distinct scores for these two dimensions of experiencing discrimination as a sexual minority. Given the nature of the constructs measured by this instrument, it was believed that effect of discrimination, as a distinct variable, would be responsible for the greatest amount of predictive variance over level of identity integration. Because resilience factors were being considered prior to perceived discrimination, severity of effect of discrimination was initially thought to be of greater salience to the individual than frequency of experience. That is, the theoretical role that resilience plays in buffering against negative experiences associated with minority status, individuals who are highly resilient were thought to be less likely to perceive experiences as discriminatory as frequently as those who are not resilient. Effect of discrimination was therefore given the 
second point of entry in the proposed regression equation, with frequency of discrimination entered as the third variable.

Finally, Frazier et al. (2004) instructed that product terms created from the standardized predictor and moderator variables be the final entries in the regression equation. In this case, this was to establish the moderating effect of resilience on perceived discrimination in predicting identity integration. Product terms were generated by multiplying together the predictor and moderator variables in their standardized form, "to represent the interaction between the predictor [perceived discrimination] and the moderator [resilience]" (p. 120). According to Frazier et al., these terms must be entered into the equation along with the standardized variables themselves because inspecting product terms alone risks confounding the effects of the moderator and predictor variables. The entire regression model is represented by the following equation:

$$
Y_{i} \text { Outness }=\left(b_{0}+b_{1} \text { Res }+b_{2} \text { Eff }+b_{3} \text { Freq }+b_{4}[\text { Res } \times \text { Eff }]+b_{5}[\text { Res } \times \text { Freq }]\right)+\varepsilon_{i}
$$

In order to test the strength and accuracy of the proposed regression model, secondary stepwise regression analyses were also conducted. According to Field (2009), the order in which variables are entered into stepwise regression equations are based solely on mathematical criteria: the predictor that has the highest simple correlation with the outcome is entered first, and the process continues with each remaining predictor variable, thereby establishing a regression model that best fits the data. For the purposes of the present analysis, both the forward method of stepwise regression and the backward method were conducted.

Final confirmatory regression analyses were conducted to determine the accuracy of conclusions drawn about outness as a process of identity integration. As noted, early 
theoretical models of sexual minority identity development were flawed in their assumption that public disclosure of identity was a necessary or important milestone of the development process. Therefore secondary regression analyses were conducted duplicating the models described above, but replacing outness as the DV with the Identity Centrality subscale of the LGBIS, which measures how central one's sexual minority identity is to their overall identity, but which does not presume public disclosure. Due to the emphasis in this study on social role functioning the OI's measure of outness was a more suitable DV for the primary analyses, but it was also considered important to investigate the ability of the regression equation to predict personal importance of one's minority identity, regardless of outness.

To investigate the second research question, additional correlational analyses were conducted to assess the nature of the relationships between the proposed IVs (resilience factors and frequency and effect of perceived discrimination) and the theoretical dimensions of LGB identity development. To establish the relationship between the proposed IVs and LGBIS subscale scores, a two-tailed bivariate correlation analysis was conducted. The purpose of this analysis was to make inferences about the role of resilience factors and perceived discrimination in overall LGB identity development. 


\section{CHAPTER THREE: RESULTS}

\section{Descriptive Statistics}

Cronbach's alpha coefficients of internal consistency were found to be good to strong for the instruments detailed below, suggesting sufficient reliability for use in the present study. Table 2 summarizes descriptive statistics for all instruments and subscales.

\section{Outness Inventory (OI)}

The OI (Fassinger \& Mohr, 2000) was used to assess participants' outness across various domains of social functioning. Responses are based on a 0 (person definitely does not know your sexual orientation status) to 7 (person definitely knows about your sexual orientation status, and it is openly talked about) scale on which participants rate the degree to which one's sexual orientation is publicly known and openly acknowledged across three domains. Each domain is represented by a distinct subscale: Out to Family $(M=5.34, S D=1.59)$, which includes parents, siblings, and extended relatives; Out to Religion $(M=1.52, S D=2.45)$, including one's religious community and religious leaders; and Out to World $(M=4.93, S D=1.59)$, including heterosexual friends, work peers, work supervisors, and strangers or new acquaintances. Averages of all three subscales are combined to form a composite score measuring Overall Outness $(M=5.07$, $S D=1.41)$. Overall Outness scores represented the dependent variable, outness, for the present study.

\section{Courage to Challenge Scale (CCS)}

The CCS (Smith \& Gray, 2009) is an instrument designed to assess hardiness in sexual minorities, and was utilized in the present study to measure the proposed moderating variable, resilience. Responses to the CCS items $(M=5.72, S D=.60)$ are 
based on a 1 (Strongly Disagree) to 7 (Strongly Agree) scale on which participants rate the degree to which they believe they are capable of enduring or persevering through negative events or difficult times.

\section{Gay and Lesbian Oppressive Situations Inventory - Frequency and Effect}

\section{(GALOSI-F \& E)}

The two GALOSI scales (Highlen, Bean, \& Sampson, 2000) were designed to measure the amount and severity of discrimination one perceives as related to one's sexual minority identity. The GALOSI-F $(M=2.30, S D=.50)$ is comprised of 49 items, whereas the GALOSI-E $(M=3.36, S D=1.08)$ is comprised of 47 items. Responses are based 1-6 scales on which participants rate the frequency and severity of effect of given statements (e.g., "I have been stereotyped based on my sexual orientation."). The GALOSI scales describe events in which sexual minorities may commonly experience discrimination. Higher scores indicate greater perceived frequency and severity of effect of discriminative experiences. Frequency and effect of discrimination were included in the present analysis as independent variables in predicting outness.

\section{Lesbian, Gay, and Bisexual Identity Scale (LGBIS)}

All eight LGBIS subscales (Mohr \& Kendra, 2011) were utilized for interpretive analyses regarding the relationships between LGB identity development and resilience as well as perceived discrimination. Participants' responses were based on a six-point Likert-type scale ranging from Disagree Strongly (1) to Agree Strongly (6). The Concealment Motivation $(M=2.59, S D=1.02)$, Identity Uncertainty $(M=1.56, S D=$ $.81)$, Acceptance Concerns $(M=2.96, S D=1.09)$, Identity Affirmation $(M=5.14, S D=$ $.78)$, Internalized Homonegativity $(M=1.66, S D=.91)$, Difficult Process $(M=2.88, S D$ 
$=.69)$, Identity Superiority $(M=1.87, S D=.85)$, and Identity Centrality $(M=4.09, S D=$ .72) were used in these analyses.

\section{Analyses}

\section{Research Question One}

The first research question asked whether resilience served to moderate levels of perceived discrimination (i.e., frequency of experience and severity of effect) in order to predict the degree to which LGB identified individuals integrate their sexual minority identities across social roles. Table 3 depicts Pearson correlations for all variables used in this analysis.

Regression analyses confirmed that resilience and frequency of perceived discriminative events (FPD) positively predicted identity integration, or outness (detailed below). However, results of the hierarchical regression analysis $(R=.36, F[5,244]=$ $7.42, p<.001)$ indicated that severity of effect of perceived discrimination (EPD) did not meaningfully or significantly contribute to the model's predictive ability, which explained $13 \%$ of the variance in outness overall (Table 4). Furthermore, the interactions between resilience and EPD and resilience and FPD (entered into the regression equation as product terms of the respective interactions) also did not significantly add to the model. This final observation is especially meaningful because it suggests that, while resilience itself is predictive of outness, it does not moderate the effects of EPD or FPD in predicting outness.

Confirmatory regression analyses confirmed the above results. In order to test the accuracy and strength of the proposed hierarchical model, both forward and backward analyses were conducted. Resilience $(R=.27, F[1,249]=19.18, p<.001)$ followed by 
resilience with $\operatorname{FPD}(R=.36, F[2,247]=18.17, p<.001)$ were the only variables entered into the forward regression, being the only two variables significantly predictive of outness (Table 5). Resilience, which was entered first, was found to explain 7\% of variance. FPD, the second and final variable entered into the forward model, contributed another $6 \%$ of variance. The two forward models explained $13 \%$ of variance in outness overall, which was consistent with the results of the initial hierarchical regression.

Results of a backward regression analysis were further supportive of the above findings (Table 6): Following the entry of all five variables $(R=.36, F[5,244]=7.42, p$ $<.001)$ into the equation, the product term for the resilience $\times$ FPD interaction was removed first $(R=.36, F[4,245]=9.25, p<.001)$, followed by the product term for the resilience $\times$ EPD interaction $(R=.36, F[3,246]=12.35, p<.001)$. Finally, EPD was removed, leaving resilience followed by FPD in the fourth and final model $(R=.36, F[2$, $247]=18.17, p<.001)$. Across all four models, explained variance remained constant at $13 \%$.

Importantly, the two product term variables, representing the interactions between resilience and FPD and resilience and EPD, were excluded from the final models in both forward and backward equations. As noted, this finding suggests that resilience does not significantly moderate the effects of FPD and EPD, as was initially proposed. Also of note, EPD, which was initially proposed to be more strongly predictive of outness than FPD, did not significantly contribute to the model, and explained approximately only $0.3 \%$ of the variance.

Also of note, a hierarchical regression analysis using identical variables and points of entry described above, was found to be a poor predictor of Identity Centrality ( $R$ 
$=.21, F[5,244]=2.14, p>.05$ ), as measured by the corresponding LGBIS subscale. The results of this model were not significant and explained only $3 \%$ of the variance in Identity Centrality. This finding is notable because (a) results indicated that Identity Centrality and outness have a moderate and significant positive relationship $(r=.37, p<$ .001 ), and (b) while Identity Centrality and outness are clearly distinct and not mutually exclusive constructs, theoretically it was expected that their development would depend upon similar processes, including resilience and experienced discrimination.

\section{Research Question Two}

The purpose of the second research question was to establish the nature of the relationship between both resilience and perceived discrimination and the eight individual theoretical dimensions of LGB identity development proposed by Mohr and Kendra (2011). Exploratory two-tailed bivariate analyses of Pearson product-moment correlations among these variables are detailed in Table 7. Overall, results were consistent with those of regression analyses, given the close established relationship between outness and identity development (Mohr \& Kendra, 2011).

Resilience was significantly correlated with seven of the eight domains. It had weak negative relationships with Concealment Motivation, Identity Uncertainty, internal homonegativity, Difficult Process, and Identity Superiority; a moderate negative relationship with Acceptance Concerns; and a moderate positive relationship to Identity Affirmation. No statistical relationship was found between resilience and Identity Centrality.

Interestingly, the two domains of perceived discrimination were far less consistently related to identity development, with both EPD and FPD relating even 
minimally to only four of the eight domains each. EPD had minimal or weak, but significant positive relationships with Concealment Motivation, Identity Uncertainty, Acceptance Concerns, and Difficult Process, and FPD had minimal but significant positive relationships with Concealment Motivation and Internalized Homonegativity. The only strong and significant positive relationships observed across all correlational analyses were found between FPD and both Acceptance Concerns and Difficult Process.

\section{Post Hoc Analyses}

Despite the failure of the proposed model to adequately predict outness, using resilience as a moderator for FPD and EPD, it was determined that the predictive nature of resilience and FPD was important to investigate further. To establish the importance of resilience and FPD in predicting outness across areas of social functioning, post-hoc forced-entry multiple regression analyses were conducted using individual sample subgroups determined by various demographic areas. Tables 8 and 9 detail Pearson product-moment correlations and regression analyses for all demographic subgroups, respectively.

Of primary interest to the present study, sexual orientation was investigated in the post-hoc analysis first. When including only responses from self-identified lesbians $(R=$ $.45, F[2,89]=11.11, p<.001)$, resilience and FPD explained $20 \%$ of the variance in outness, which was contrasted by $12 \%$ of explained variance in outness among gay men $(R=.35, F[2,103]=7.07, p<.01)$ for whom FPD did not significantly contribute to the

model. Among bisexuals $(R=.27, F[2,49]=1.89$, ns $)$, the model explained only $7 \%$ of the variance in outness, and was not statistically significant.

Difference in predicted outness between genders was also investigated. Among 
female respondents $(R=.40, F[2,135]=12.80, p<.001)$, resilience and FPD predicted $16 \%$ of the variance in outness, twice as much as the $8 \%$ of explained variance found among male respondents $(R=.28, F[2,102]=4.27, p<.05)$ for whom FPD was not a significant predictor of outness. Although a small number of self-identified transgender individuals, as well as other gender identities, responded to the survey, response rates were too low for this level of analysis.

Age was considered next. Responses were grouped into three categories based on age: $18-29,30-45$, and 46-66. Regression analyses demonstrated that for the youngest group $(R=.31, F[2,110]=5.68, p<.01)$ resilience accounted for $9 \%$ of the variance in outness, though FPD did not significantly contribute to the model. This was similar to the $8 \%$ of explained variance of the middle group $(R=.29, F[2,74]=3.39, p<.05)$, although resilience did not significantly contribute to the model for these individuals. Explained variance of the oldest group $(R=.43, F[2,57]=6.56, p<.01)$, however, was found to be much higher at $19 \%$, and were the only age group for whom both resilience and FPD were significant predictors.

In order to investigate the influence of racial identity, two categories were created based on self-identified race. Although categorization of individual racial or ethnic groups would have likely yielded important information about resilience and discrimination across cultures, due to the largely homogenous racial/ethnic makeup of the present sample two groups were created: individuals who self-identified as non-Hispanic White or Caucasian, and other non-White racial/ethnic minority groups. In White respondents $(R=.37, F[2,199]=15.46, p<.001), 13 \%$ of the variance in outness was explained by resilience and FPD. Although explained variance in outness for non-White 
respondents $(R=.41, F[2,45]=4.56, p<.05)$ was slightly higher at $17 \%$, the $F$ ratio was significantly smaller and, notably, FPD did not contribute significantly to the model.

Similarly, two categories were created to group respondents based on their selfidentified religious orientation: those who reported participation in a religious community, and those who did not (including self-identified agnostics, atheists, and those who endorsed no religion). Regression analyses indicated that resilience and FPD were slightly more predictive of outness in those who identified themselves as religious $(R=$ $.42, F[2,98]=10.25, p<.001)$ compared with those who $\operatorname{did} \operatorname{not}(R=.36, F[2,116]=$ $8.48, p<.001)$. For religious individuals the model predicted $17 \%$ of variance in outness and $13 \%$ for those who were not.

Categories were also created in order to group respondents based on relationship status. Those individuals who reported involvement in committed relationships (i.e., respondents who were reportedly currently dating; monogamously coupled; and civilly joined or married) were included in the first group. The second group consisted of respondents who endorsed being single, separated or divorced, or widowed. For individuals who reported having a romantic partner in their lives presently $(R=.38, F[2$, $156]=13.47, p<.001)$, resilience and FPD explained $15 \%$ of the variance in outness, as compared to $8 \%$ for those who did not $(R=.29, F[2,81]=3.64, p<.05)$.

Finally, level of education was investigated. Again, responses were categorized into subgroups based on reported level of highest completed education: (1) individuals who had completed high school as well as individuals who had completed some college or an associates degree, (2) individuals who had completed college (i.e., bachelors degree) and, (3) individuals who reported graduate level education (e.g., masters or 
above). Regression analyses suggested that among lower educated individuals $(R=.25$, $F[2,58]=1.86, p>.05)$, resilience and FPD did not significantly predict variance in outness. Predicted variance in outness for college educated respondents $(R=.48, F[2$, $59]=8.64, p=.001$ ) was found to be $23 \%$ with only resilience contributing significantly to the model, and for masters- and doctoral-level respondents $(R=.35, F[2,122]=8.41$, $p=.001)$, resilience and FPD predicted $12 \%$ of the variance. 


\section{CHAPTER FOUR: DISCUSSION}

There were two primary purposes for this study. One was to examine the influence of resilience on the identity integration process of sexual minorities. Specifically, the first purpose was to determine whether resilience functions as a buffering agent against the negative effects of perceived discrimination, such that individuals who reported high levels of resilience would be less likely to experience these negative effects. The second purpose of this study was more exploratory in nature. Previous research has identified multiple theoretical domains of sexual minority identity development (Fassinger \& Miller, 1996; Mohr \& Kendra, 2011), and the role of outness in identity development and integration has been established (Mohr \& Fassinger, 2000). The second purpose was to extend this line of research by establishing the relationships of these theoretical constructs to observed internal and external processes that may influence their development. The research questions guiding this study asked about the roles of, and relationships between, these different variables in sexual minority identity integration. This chapter discusses the findings and implications of the preceding research, and suggests answers to the research questions. Also discussed are limitations of the present study and directions for future research.

\section{Findings and Implications}

\section{Research Question 1}

The first research question asked whether resilience moderated the overall effects of perceived discrimination in predicting outness in lesbian, gay, and bisexual adults across domains of social functioning. It was thought that the internal and external characteristics associated with resilience, which have been implicated in the positive 
development of minority identities broadly (Kwon, 2013), would be protective against the deleterious effects of experienced discrimination (including severity of effect and perceived frequency).

Statistically, it was proposed that the product terms created from the interactions between resilience and effect of perceived discrimination $(\mathrm{R} \times \mathrm{EPD})$ and resilience and frequency of perceived discrimination $(\mathrm{R} \times \mathrm{FPD})$ would contribute meaningfully and significantly to a regression model for predicting identity integration. In order to account for the external (social) as well as internal (private) manifestations of identity integration, two criterion variables were tested in analyses. The first, outness, is the degree to which one's sexual minority identity is publicly known and openly acknowledged across social contexts (Mohr \& Fassinger, 2000), and was the primary criterion variable for the present study. The second, Identity Centrality, is a construct developed by Mohr and Kendra (2011) measuring the degree to which one's minority identity is important to one's overall identity, but which does not load heavily on public disclosure of said identity. This was incorporated for confirmatory purposes, in order to acknowledge that sexual minority identity integration may occur, regardless of whether one chooses to "come out."

Hierarchical regression analyses suggested that resilience in fact does not serve to moderate severity of effect and frequency of perceived discrimination in predicting identity integration. Rather than contributing to and ideally surpassing the predictive ability of the independent variables, the product terms described above did neither: indeed, the interaction effects between resilience and both EPD and FPD were found to be poor overall predictors of identity integration (both outness and Identity Centrality). 
Additionally, EPD, which was initially hypothesized to be an important predictor variable (second only to resilience, and entered into the original regression equation accordingly), was also found to be minimally related to identity integration, and was a similarly poor contributor to the model's predictive ability. Subsequent forward and backward regression analyses confirmed these results: all confirmatory regression analyses concluded with the exclusion of both product terms $(\mathrm{R} \times \mathrm{EPD}$ and $\mathrm{R} \times \mathrm{FPD})$ as well as EPD due to these variables' lack of meaningful contribution to the respective model's predictive ability.

The main result of the hierarchical regression; that resilience does not serve to moderate or buffer the effects of discrimination as hypothesized; differed from expectations based on previous reports. In his review of resilience among LGB community members, Kwon (2013) suggested that evidence exists in support of Cohen's (2004) assertion that people generally appraise stressful events more positively when they have increased access to positive social support. Specifically, Kwon argued that this mechanism would work among LGB individuals to generally lower reactivity to prejudice in the community. The buffering effect of resilience against discrimination, according to Kwon's argument, was precisely what was expected of the current findings, but was not observed.

Despite the failure of the proposed regression model to predict outness, some interesting results were still obtained. Specifically it was found that resilience, functioning as an independent variable rather than a moderator, followed by FPD were together significant predictors of outness, explaining roughly $13 \%$ of total variance. This finding is notable for two reasons. First, it suggests that resilience may not have the 
buffering effect against minority stress among this population that has been proposed in some previous literature (Kwon, 2013), but rather may function independently as a predictive factor, regardless of the effects of minority stress. Second, it suggests that frequency of perceived discrimination is more critical and deleterious to the overall development of sexual minority identity than perceived severity of effect. This is notably contradictory to what was initially hypothesized: It was presumed that EPD would ultimately be more significant in predicting outness in that negative effects of discrimination were expected to be more highly resistant to the protective nature of resilience.

The lack of the hypothesized significance of EPD as a predictor may be explained by the recent literature on microaggression and related constructs. Much of this literature has positioned frequency of discriminative events as being crucially linked to one's minority identity and associated minority stress, regardless of effect (Sue et al., 2007). That is, according to this literature, one must not underestimate the significance of the "daily hassles" that arguably occur regularly as the result of one's minority status, despite the fact that many of these events often appear innocuous or unimportant when taken by themselves (Swim et al., 2009). It has been suggested that the cumulative result of the frequency of such events, however, can have substantial bearing on one's self-assessment of one's minority identity (Sue et al.).

\section{Research Question 2}

The second research question was exploratory in nature, and was concerned with the quality of the relationships between the independent variables of the hierarchical regression model above and the eight subscales of the Lesbian, Gay, and Bisexual 
Identity Scale (LGBIS; Mohr \& Kendra, 2011). The LGBIS is widely used in the literature as a measure of sexual orientation identity, and the original version was developed alongside the Outness Inventory from the same data (OI; Mohr \& Fassinger, 2000). One advantage of the LGBIS is the breadth and scope of its theoretical foundation in defining sexual minority identity, as represented by the eight theoretical domains of identity development. Due to the present study's focus on identity integration, which relates to a theoretical assumption about final stages of LGB identity development, it was considered important to investigate the relationship of the proposed predictor variables to identity overall.

Many relationships performed as would be expected. For example, FPD was highly related to Difficult Process (measuring the personal challenges associated with identifying as LGB) and Acceptance Concerns (measuring fear or worry associated with being stigmatized as a sexual minority). These findings are consistent with earlier reports on the negative effects of stigma and discrimination (experienced or anticipated) on LGB identity formation. (See, for example, White and Stephenson [2014], who found that experience of discrimination was associated with greater intrapersonal conflict related to sexual orientation and increased engagement in risky sexual behavior.) There were, however, notable exceptions. For instance, Identity Superiority, which essentially measures the degree to which sexual minorities favor other LGB individuals to heterosexuals and represents a positive (if perhaps overly positive) view of sexual minority identity, had a significant negative relationship to resilience. Also unexpected was the generally weak relationship that EPD shared with all LGBIS subscales, given the instrument's heavy focus on challenges and stress associated with LGB identity 
development.

Perhaps most notably, however, Identity Centrality, which was developed to measure the degree to which one's sexual minority identity was important to overall identity (and therefore theoretically of most interest to the present study), was found to be the only LGBIS subscale essentially unrelated to the three predictor variables, resilience, EPD, and FPD. This is surprising because the four items on this subscale all address to some degree how strongly respondents identify as LGB (e.g., "I believe being LGB is an important part of me"). Assuming that some measure of stress may be associated with membership in any minority group at one time or another, it was expected that those individuals who reported strong identification as LGB would also report at least moderate levels of resilience. Regardless of outness, which was expected to be clearly related to the experience of discrimination, resilience was expected to be positively related to the subscales measuring the strength of one's personal identification as LGB. By contrast Identity Affirmation, which is thought to measure the positive feelings one has about one's own sexual minority identity, was not only the single LGBIS subscale positively related to resilience, it was also the subscale with the strongest relationship to resilience. Indeed, resilience was significantly related to all subscales except Identity Centrality.

The meaning of the unexpected lack of correlation between resilience and Identity Centrality is not clear. Resilience as a theoretical construct has been widely considered in the literature and a concrete definition has not yet been established. Even the most restrictive definitions, however, typically acknowledge that resilience is driven by internal and/or external characteristics that promote strength and adaptability in the face of challenges and barriers to growth (e.g., Herrick et al., 2013). It is surprising, then, that 
according to the present results resilience seems to have little to do with the development of a sexual minority identity that is central and integral to one's overall identity.

One possible explanation for this finding is that development of a centralized LGB identity is not dependent upon one's ability to adapt to challenges or demonstrate strength in response to adversity. Perhaps the more "positive" psychosocial characteristics commonly associated with resilience are largely unrelated to this process. As noted, among the LGBIS subscales, resilience was most strongly related to Identity Affirmation, which loads more clearly on the "positive" thoughts and feelings one may associate with resilience (e.g., pride) that are absent from the Identity Centrality items. The present results suggest that the assumption that positive feelings regarding one's selfidentity would be related to the personal importance of that identity may be erroneous. That is, such an assumption can undermine or minimize the complex feelings people may have about identifying as LGB, even when that aspect of their identity is central to how they see themselves.

\section{Post Hoc Analyses}

Perhaps the most interesting results of the present study were derived from post hoc analyses. These were completed by rerunning the regression equation established to be most predictive of outness (including resilience first, followed by FPD), while including only members of specific subgroups determined by demographic self-report. Subgroups based on sexual orientation (lesbian, gay, and bisexual); gender (female and male); age (18-29, 30-45, and 46-66+); race/ethnicity (White and non-White); religion (religious and not religious); relationship status (partnered and not partnered); and level of education (below bachelors level, bachelors level, and above bachelors level including 
masters and doctoral level) were all investigated. Results were mostly consistent with the extant resilience literature (Cohen, 2004; Dentato, Orwat, Spiro, \& Walker, 2014; Kwon, 2013; Saewyc, 2011), in that in most groups greater variance in outness was predicted by resilience and discrimination based on cultural variables, available social support, and education.

Sexual Orientation. Regarding sexual orientation, the most striking finding is the range of variance explained in outness across the subgroups of lesbians, gay men, and bisexuals. Lesbian women are the only group for whom resilience and FPD were both significant predictors of outness, which was estimated to account for approximately $20 \%$ of the variance. This figure is about twice as much as the explained mean variance between gay men and bisexuals. Additionally, for gay men, only resilience significantly contributed to the regression model, and neither resilience nor FPD significantly predicted outness in bisexuals.

A number of interesting observations can be made based on these findings. First, it is possible that the dual minority status of female lesbians (gender and sexual orientation) results in more challenges associated with coming out, with outness apparently more dependent on both resilience and the frequency with which discriminative experiences occur. Notably, the effect of gender alone does not explain the discrepancy in variance across sexual orientations, because the bisexual group was composed of approximately $77 \%$ women. If gender, and not sexual orientation, were solely responsible for explaining the difference in variance for lesbians compared to the other groups, it would be expected that more of the variance in outness would be explained in the bisexual subgroup as well. By contrast, gay men reported less FPD in 
general, resulting in discrimination being less predictive of outness for this group overall.

Finally, the failure of both variables to predict outness in bisexuals is interesting as well, in that it contradicts to some degree existing ideas about bisexuals as having "double bind" minority status. That is, it has been suggested that bisexuals often feel non-normative in both homosexual groups and heterosexual groups, often resulting in bisexuals reporting increased discrimination from both groups (Bostwick, Boyd, Hughes, West, \& McCabe, 2014). Based on these earlier assertions, it would have been expected for one or both of the independent variables to predict outness, as it does for the other subgroups. The fact that they do not may suggest one of two things: (1) despite not having as large a sexual orientation subgroup of their own, bisexuality may be a slightly "safer" sexual minority identity in that engagement in more culturally normative sexual practices may be evaluated more favorably by majority groups; or (2) given the distinct differences in sexuality among homosexuals and bisexuals, it is likely that the coming out process across the two groups is inherently dissimilar. Including bisexuals in the analysis may represent a limitation of the present study.

Gender. The findings related to gender are consistent with those reported above for sexual orientation. Approximately $16 \%$ of variance in outness was explained by resilience and FPD for women. It is important to note that approximately $65 \%$ of female participants self-identified as lesbian, which likely contributed to much of the residual variance in outness explained for this group. Despite this, beta coefficients and respective significance levels for resilience and FPD remained consistent for females as compared to the lesbian group, and the $F$-ratio of the equation actually improved, suggesting the finding of explained variance for females is independent of the effects of 
sexual orientation.

For male respondents, only resilience significantly predicted outness, which was also consistent with the above sexual orientation results. Due to low response rate for transgender individuals, this group was excluded from the present analysis. Though this group would certainly represent gender minorities in addition to women, due to their exclusion the hypothesis of the "dual minority" effect as it relates to gender and outness can only be speculated. Certainly, the consistency of the available findings between gender and sexual orientation analyses compellingly suggests that resilience and discrimination are more salient for those individuals who hold minority status in more than one group.

Age. Another dimension of minority identity's influence on outness is the cohort effect. For those participants who entered early adulthood between approximately 1966 and 1986, more than twice as much of the variance in outness (19\%) was explained by resilience and FPD than for the younger cohorts (8-9\%). While no less interesting, this finding was expected and is consistent with existing knowledge of differences across cohorts of sexual minorities (Dentato et al., 2013; King \& Orel, 2012). In the context of the sociopolitical climates of the years when most or all of the older group would have likely been undergoing their LGB identity development process, it is likely that these individuals experienced increased levels of discrimination. Indeed, FPD was most strongly related to this age group as compared to younger respondents $(r=.36, p<.01)$. Additionally, members of this group may have been more reliant than their younger counterparts on sources of strength in the face of adversity during these times. The only other respondents for whom resilience significantly predicted outness were in the 
youngest group, which also makes sense: sociopolitical context notwithstanding, the LGB identity development process is commonly seen as a difficult a period for young people, who similarly may rely on internal and external sources of strength to navigate it, regardless of levels of discrimination.

Race/Ethnicity. The results of the regression analysis controlling for race/ethnicity deviated from expectations, and do not clearly support the "dual identity" hypothesis of minority stress suggested by findings cited above. An important note is that this analysis is inherently problematic in that multiple racial minority subgroups have been forced into a single group, delineated only by its members' non-White status. This was necessary due to the substantial lack of responses from individual racial and ethnic groups other than White, and was completed for the present analysis only for the purpose of examining further the effect of multiple minority identities on outness (in the case of this analysis, non-White would by definition constitute a racial/ethnic minority identity). This result should be interpreted with caution, as it is assumed that the influence of one's relative racial/ethnic minority status on his or her overall minority identity would vary greatly across subgroups, and depend upon such other variables as age, generational status, gender, SES, and regional considerations.

Such as they are, the present results did not reveal as striking a difference in explained variance between groups as might have been expected, with only about $4 \%$ more of variance in outness explained for non-Whites (17\%) than Whites (13\%). Interestingly, FPD did not contribute significantly to the regression analysis and was found to have no remarkable relationship to outness for this group $(r=-.16, \mathrm{~ns})$. By contrast, FPD did significantly contribute to the model's predictive ability for White 
respondents. Of note, however, for non-White respondents resilience had a strong significant relationship to outness $(r=.41, p<.01)$ and predicted nearly all of its variance.

Given the problematic nature of this analysis noted above, it is difficult to interpret the meaning of this finding. It is possible, however, that minority identity development for individuals who identified as members of minority groups from birth may have more experience with overt or covert levels of discrimination than their White counterparts. This suggestion may explain two of the notable findings of this analysis: (1) White respondents may have had less exposure to discrimination across the lifespan, resulting in increased sensitivity to its frequency once the LGB identity development process had begun later in life; and (2) racial/ethnic minorities may have developed as more highly resilient than their White counterparts as a result of their experiences as minorities prior to the LGB identity development process, making resilience an important part of their minority-stress management in general.

Religion. Given the theoretical importance of social support in the development of resilience (Cohen, 2004), the results of analyses based on religious participation were consistent with expectations. For individuals who reported participation in a formal religious or spiritual community, resilience and FPD explained 17\% of the variance as compared to $13 \%$ in those who denied religious activity. As noted, the literature has clearly established the importance of a supportive community and meaningful social interaction as important to the development of a resilient approach to coping with challenges, which may explain the observed difference in explained variance between these two groups. 
It is interesting to note, however, that for more religiously active LGB individuals, FPD contributed more strongly and significantly to the model suggesting that this group is also more sensitive to discrimination than their non-religious peers. One clear possibility for this is that mainstream Western religions have traditionally portrayed same-sex sexual behaviors negatively (Walker \& Longmire-Avital, 2013), and discrimination related to LGB identity experienced within religious communities may diminish the protective effect of social support and contribute to increased sensitivity to FPD. Although other minority groups (e.g., racial and ethnic minorities) have been shown to historically turn to religious faith as a source of resilience-consistent strength and coping (in the form of increased hope and ability to persevere), the same may not be true for the LGB population. Walker and Longmire-Avital (2013) noted potentially deleterious effects of religious involvement in LGB young adults, and indeed noted that a marked absence of religious faith was associated with increased ability to cope with adversity.

Relationship Status. Another dimension of social support includes romantic partnership. As with the findings noted above, the results of these analyses were consistent with expectations in terms of variance in outness, nearly twice as much of which was explained in respondents who reported romantic partnership (15\%) as compared to those who did not (8\%). As with religion, however, resilience did not contribute to the model as meaningfully as FPD for those individuals who reported the additional support. Similar to what was reported above, this finding is equally interesting because it suggests an increased vulnerability to FPD not commonly accounted for in theories of resilience in the LGB population. Namely, it is thought that perhaps this 
finding can be at least partly explained by the risk of inadvertently disclosing one's LGB identity among the general population when appearing in public with one's romantic partner.

As described in Chapter 1, coming out is typically considered a continuous process, one that LGB individuals must consider with each new acquaintance or social context. The present findings regarding romantic partnership are suggestive of this dilemma, in that LGB individuals who appear in the community may still retain some control over the public nature of their sexual minority identity. Those who appear with their partners, by contrast, may risk outing themselves in contexts in which they would not normally chose to do so, possibly resulting in increased frequency of discriminative events. In fact, there is some available literature that supports this interpretation: according to Knoble and Linville (2012), "increased visibility as a GLB person and samegender couple increased [one's] experience of gay-related stress," (p. 337).

Education. Perhaps the most unexpected discrepancy in variance across post hoc analysis subgroups was found among education level. According to the present results, resilience and FPD predicted significantly more variance in outness among college graduates (i.e., bachelors level), then they did in individuals who had a lower than bachelors-level education (i.e., high school or associates level), or those who have completed graduate level education (i.e., masters or doctoral level). For college graduates, the regression model explained $22 \%$ of the variance in outness as compared to the $12 \%$ for post-baccalaureate educated individuals. The model failed to significantly predict outness for individuals who had less than a bachelors-level education.

Explanation for these differences can only be speculated at, though they may have 
as much to do with sampling effects as any characteristics inherent to the groups themselves. That is, a large portion of the present sample was recruited from university settings, and is clearly comprised of individuals educated to a degree that is likely not generalizable to the general LGB population; for instance, there are nearly as many masters and doctoral level respondents in the total sample as any other level of education. Given these demographics, it is possible that current results can be explained in part by literature suggesting that education level is associated with greater resilience (Frankenburg, Sikoki, Sumantri, Suriastini, \& Thomas, 2013). Even so, that information would not necessarily explain the discrepancy between the mid and high levels of education and there is a surprising paucity of current literature related to resilience in LGB-identified individuals in university settings.

Perhaps current results can also be explained in part by extant educational psychology literature related to resilience and school connectedness. It has been previously reported that at-risk students who feel supported in academic environments are more likely to succeed than their unsupported peers (e.g., Furlong, Sharkey, Quirk, \& Dowdy, 2011), but this literature is focused primarily on pre-collegiate settings and so may not fully capture these current findings. Regardless, given the high rates of respondents associated with university-based LGB community centers, it may be assumed that many of them were enrolled students at the time of participation (either at undergraduate or graduate levels). This may suggest that access to academic support as well as university-based community support broadly, both of which are associated with resilience, would likely be higher for this group of respondents. 


\section{Limitations}

Some of the primary limitations of this study have already been suggested earlier in the chapter, including the unusually high reported level of education of respondents. Issues related to sampling and participant self-selection are common to LGB research in, and limit the generalizability of, results to the general LGB population. Broadly speaking, given the "invisible" nature of low-identified LGB individuals or those who elect not to disclose their LGB identity publicly, it is difficult to engage this group in research of this kind. Rather it is common in the literature, as was done for the present study, to recruit participants from university- and community-based LGB support centers as well as online listservs. It is unlikely that individuals who are actively questioning their sexual orientation or have decided not to come out for personal reasons would be associated with any of these forums or resources for LGB community involvement.

Therefore, as was acknowledged in Chapter 2, respondents for the present study were generally highly identified and openly LGB, as was expected. Although results did not necessarily demonstrate hypothesized relationships between outness, resilience, and discrimination, it is possible that the highly identified nature of this sample skewed results. As such, it may be that any or all of the predictor variables were less salient to the present sample due to lower current identity-related stress than would be noted in respondents who were experiencing greater internal or external conflict related to the LGB identity development process.

More specific sampling issues were noted as well. Primarily, the use of internetbased snowball sampling resulted in little control over the nature and types of responses this study attracted. Initially, it was planned that direct communication only with 
community-based LGB centers would yield the necessary sample. When it became apparent that augmentative sampling would be necessary to improve response rates, university-based LGB centers were also contacted. Most crucially, however, the use of internet-based social networking (specifically via Facebook) was incorporated. Due to the mechanics of this method of communication, including rapid and largely untraceable dissemination of information, there was little control over whom the study reached. Once the link to the online survey was posted on pages (or "walls") managed by target LGB centers, it was impossible to follow its progress across the Internet. Anecdotally, the effect of this was noted in an initial review of results: despite being well publicized as a study of sexual minority identity a small number of respondents reported their sexual orientation as "heterosexual." These responses were naturally excluded from analysis, but it demonstrates the lack of control over who was free to submit responses on the survey, and therefore may call into question the veracity of the anonymous data that were included in analyses.

Regarding limitations of the statistical analysis, forward stepwise regression, the analysis utilized primarily in the present study in the post hoc analyses, has been reported to have limited replicability (Menard, 2002). More specifically, results of stepwise analyses are highly influenced by sampling error, such that random variations in the collected data are reflected in the results. According to Menard (2002), these variations are difficult to replicate due to their idiosyncratic nature, and therefore conclusions drawn based on findings are sometimes difficult to generalize to a greater population.

\section{Future Directions}

Generally speaking, resilience research has tended to identify a wide range of 
variables that may (or may not) need to be in place in order for resiliency to successfully develop, including personal characteristics (e.g., self-efficacy) and environmental variables (e.g., social support). These variables can often seem only loosely related, and so triangulation of contributing variables toward greater construct specificity is still needed. One of the initial intentions behind the present study was to establish more firmly the role of internal variables in the development of resilience. To that end, the instrument selected for measurement of resilience (CCS; Smith \& Gray, 2009), was one that emphasized, in terms of item content, personal characteristics that respondents believed were associated with their relative hardiness. The failure of the present results to firmly establish a moderating effect of resilience on experience of discrimination in predicting outness may suggest that future research should focus more intently on the external variables that may contribute to development of resilience.

Furthermore, research into the roles of resilience and discrimination in the LGB identity development process may also be warranted. As noted, the present sample was generally highly LGB identified. Individuals who have established both internal (i.e., positive self-identity) and external (i.e., membership in a supportive community) factors supportive of identity integration and outness are therefore less in need of intervention to facilitate their development process. Given the complex nature of LGB identity development, there are presumably countless individual factors that may support or discourage one's personal growth through this process. The aim of this cross-sectional study was to offer insight into the continued importance and effect of resilience and discrimination in LGB-identified individuals. Retrospective or longitudinal studies, which investigate earlier stages of LGB identity process, may be useful to future research 
in order to more clearly establish the roles of these specific variables when individuals are more actively going through the process of solidifying their identities as sexual minorities.

\section{Conclusion}

McCarn and Fassinger's (1996) model of sexual minority identity development served as the theoretical framework for this study. According to the authors, the final stage of identity development occurs when one's sexual minority identity has been integrated, either publicly or privately, in one's overall identity. The results of the present study contribute to the literature that has followed McCarn and Fassinger in demonstrating that the identity integration process is variable across domains; indeed even among the present sample, a group found to be highly LGB-identified, less than 5\% of respondents endorsed being completely out in all current social contexts. The present study has also added to this literature by further highlighting some of the specific variables that, individually and in tandem, may serve to both facilitate as well as discourage the identity development process.

The integration of sexual minority identity status into one's overall identity is clearly a very personal and individualized process. Certainly this study and others can help point researchers and clinicians to specific variables that may put individuals at greater risk or serve to provide strength. Given the unique nature of this process across individuals, however, it is important to acknowledge that patterns observed in research might prove misleading on a case-by-case basis. As such, it is important that clinicians working with this population avoid assumptions about psychosocial variables commonly regarded as positive that may be sources of stress. Conversely, over-pathologizing 
sociocultural involvement presumed to be stress inducing must also be avoided, and these variables must be examined for their strength-giving potential.

The function that counseling psychology may serve in this is to support the knowledge and understanding within psychology at large, highlighting the importance of strength-based approaches in addition to those that focus chiefly on dysfunction. As counseling psychologists, it is critical to maintain a careful understanding across groups and subgroups of those variables that may protect against such negative outcomes as mental and physical illnesses; poor inter- and intrapersonal functioning; risk behaviors such as drug use or unsafe sex practices; and suicidality. In addition, we can direct the conversation beyond symptom reduction as a primary goal of intervention, and which naturally follows overemphasis on pathology, and continue to suggest preventative approaches that may lead not just to functioning, but also to flourishing. It is hoped that this study in some way contributes to this conversation.

Over the course of the previous five decades and earlier, LGB identity development has been the focus of a significant amount of social, scientific, media, and political scrutiny and messages about the process are varied and often strong. Ultimately, it is hoped that the present study serves to further the field's understanding of a relatively normative developmental process with which increased intrapersonal and environmental stress is often associated. Despite the stressors associated with the LGB-identity development process, however, it is clearly possible to overcome them and for integration of sexual minority identity to take place. The goal of the present study was to contribute to the understanding of what facilitates this process, even across one's diverse and often changing social environment. It is my sincerest hope that this goal was achieved. 


\section{References}

American Psychiatric Association (1980). Diagnostic and statistical manual of mental disorders (3rd ed.). Washington, DC: Author.

American Psychological Association. (2012). Guidelines for psychological practice with lesbian, gay, and bisexual clients. American Psychologist, 67(1), 10-42.

Anderson A. L. (1998). Strengths of gay male youth: An untold story. Child and Adolescent Social Work Journal, 15(1), 55-71.

Anderson, E. (2002). Openly gay athletes: Contesting hegemonic masculinity in a homophobic environment. Gender and Society, 16(6), 860-877.

Baker, F. D. (2008). The interrelatedness of homosexual identity development and perceptions of campus climate for gay, lesbian, bisexual, and transgender students at the University of South Florida, Tampa campus (Doctoral dissertation, UNIVERSITY OF SOUTH FLORIDA). Retrieved from http://scholarcommons.usf.edu/etd/127

Balsam, K. F., \& Mohr, J. J. (2007). Adaptation to sexual orientation stigma: A comparison of bisexual and lesbian/gay adults. Journal of Counseling Psychology, 54(3), 306-319.

Becker, A. B. (2014). Employment Discrimination, Local School Boards, and LGBT Civil Rights: Reviewing 25 Years of Public Opinion Data. International Journal of Public Opinion Research, edu003.

Bilodeau, B. L., \& Renn, K. A. (2005). Analysis of LGBT identity development models and implications for practice. New Directions for Student Services (111), $25-39$. 
Bostwick, W. B., Boyd, C. J., Hughes, T. L., West, B. T., \& McCabe, S. E. (2014).

Discrimination and mental health among lesbian, gay, and bisexual adults in the United States. American Journal of Orthopsychiatry, 84(1), 35-45.

Bowleg, L., Huang, J., Brooks, K., Black, A., \& Burkholder, G. (2003). Triple jeopardy and beyond: Multiple minority stress and resilience among Black lesbians. Journal of Lesbian Studies, 7(4), 87-108.

Brewster, M. E., Velez, B., DeBlaere, C., \& Moradi, B. (2012). Transgender individuals' workplace experiences: The applicability of sexual minority measures and models. Journal of Counseling Psychology, 59(1), 60-70.

Broido, E. M. (2000). Constructing identity: The nature and meaning of lesbian, gay, and bisexual identities. In R. M. Perez, K. A. DeBord, \& K. J. Bieschke (Eds.), Handbook of counseling and psychotherapy with lesbian, gay, and bisexual clients (pp. 13-34). Washington, DC: American Psychological Association.

Brown, L. S. (2005). The neglect of lesbian, gay, bisexual, and transgendered clients. In J. C. Norcross, L. E. Beutler, \& R. F. Levant (Eds.), Evidence-based practices in mental health: Debate and dialogue on the fundamental questions. Washington, DC: American Psychological Association.

Burks, D. J. (2011). Lesbian, gay, and bisexual victimization in the military: An unintended consequence of “Don't Ask, Don't Tell”? The American Psychologist, 66(7), 604-613.

Cass, V. C. (1979). Homosexual identity formation: A theoretical model. Journal of Homosexuality, 4, 219-235

Cass, V. C. (1984). Homosexual identity formation: Testing a theoretical model. 
Journal of Sex Research, 20, 143-167.

Cochran, S. D. (2001). Emerging issues in research on lesbians' and gay mens' mental health: Does sexual orientation really matter? American Psychologist, 56(11), 931-947.

Cohen, S. (2004). Social relationships and health. American Psychologist, 59, 676-684.

Costign, C. L., Koryzma, C. M., Hua, J. M., \& Chance, L. J. (2010). Ethnic identity, achievement, and psychological adjustment: Examining risk and resilience among youth from immigrant Chinese families in Canada. Cultural diversity and ethnic minority psychology, 16(2), 264.

Creed, W. E. D., DeJordy, R., \& Lok, J. (2010). Being the change: Resolving institutional contradiction through identity work. Academy of Management Journal, 53(6), 1336-1364.

D’Augelli, A. R. (1994). Identity development and sexual orientation: Toward a model of lesbian, gay, and bisexual development. In E. J. Trickett, R. J. Watts, and D. Birman (eds.), Human Diversity: Perspectives on People in Context. San Francisco: Jossey-Bass.

Dentato, M. P., Orwat, J., Spira, M., \& Walker, B. (2014). Examining cohort differences and resilience among the aging LGBT community: Implications for education and practice among an expansively diverse population. Journal of Human Behavior in the Social Environment, 24(3), 316-328.

Division 44/Committee on Lesbian, Gay, and Bisexual Concerns Joint Task Force on Guidelines for Psychotherapy with Lesbian, Gay, and Bisexual Clients. (2000). Guidelines for psychotherapy with lesbian, gay, and bisexual clients. American 
Psychologist, 55, 1440-1451.

Eldridge, J., \& Johnson, P. (2011). The relationship between old-fashioned and modern heterosexism to social dominance orientation and structural violence. Journal of Homosexuality, 58, 382-401.

Fassinger, R. E. (2000). Applying counseling theories to lesbian, gay, and bisexual clients: Pitfalls and possibilities. In R. M. Perez, K. A. DeBord, \& K. J. Bieschke (Eds.), Handbook of counseling and psychotherapy with lesbian, gay, and bisexual clients (pp. 107-132). Washington, DC: American Psychological Association.

Fassinger, R. E., \& Miller, B. A. (1996). Validation of an inclusive model of sexual minority identity formation on a sample of gay men. Journal of Homosexuality, 32(2), 53-78.

Feldman, S. E. (2012). The Impact of Outness and Lesbian, Gay, and Bisexual Identity Formation on Mental Health (Doctoral dissertation, Columbia University).

Field, A. (2009). Discovering statistics using SPSS. Sage Publications Limited.

Frankenberg, E., B. Sikoki, C. Sumantri, W. Suriastini, and D. Thomas. (2013) Education, vulnerability, and resilience after a natural disaster. Ecology and Society, 18(2), 16.

Frasier, P. A., Tix, A. P., \& Barron, K. E. (2004). Testing moderator and mediator effects in counseling psychology research. Journal of Counseling Psychology, 51(1), $115-134$.

Frosch, D. (2013, June 29). In support groups for gay military members, plenty of asking 
and telling. The New York Times, p. A23

Furlong, M., Sharkey, J., Quirk, M., \& Dowdy, E. (2011). Exploring the Protective and Promotive Effects of School Connectedness on the Relation between Psychological Health Risk and Problem Behaviors/Experiences. Journal of Educational \& Developmental Psychology, 1(1), 18-34.

Gates, G. J. (2011). How many people are lesbian, gay, bisexual, and transgender? The Williams Institute. Retrieved from www.law.ucla.edu/williamsinstitute.

Gonsiorek, J. (1991). The empirical basis for the demise of the illness model of homosexuality. In J. Gonsiorek \& J. Weinrich (Eds.) Homosexuality: Research implications for public policy (pp. 115-136). Newbury Park, CA: Sage.

Herrick, A. L., Lim, S. H., Wei, C., Smith, H., Guadamuz, T., Friedman, M. S., \& Stall, R. (2011). Resilience as an Untapped Resource in Behavioral Intervention Design for Gay Men. AIDS and Behavior, 15, S25-S29.

Herrick, A. L., Stall, R., Goldhammer, H., Egan, J. E., \& Mayer, K. H. (2013). Resilience as a research framework and as a cornerstone of prevention research for gay and bisexual men: Theory and evidence. AIDS and Behavior. Retrieved from link.springer.com.

Highlen, P. S., Bean, M. C., Sampson, M. G. (2000, August). Preliminary development of the Gay and Lesbian Oppressive Situations Inventory - Frequency and Effect $(G A L O S I-F \& E)$. Paper presented at the annual meeting of the American Psychological Association, Washington, D.C.

Jones, L., \& MacCarthy, M. (2010). Mapping the landscape of gay men's football. Leisure Studies, 29(2), 161-173. 
Kahn, M. J. (1991). Factors affecting the coming out process for lesbians. Journal of Homosexuality, 21, 47-70.

King, S. D., \& Orel, N. (2012). Midlife and older gay men living with HIV/AIDS: The influence of resiliency and psychosocial stress factors on health needs. Journal of Gay and Lesbian Social Services, 24, 346-370.

Knoble, N. B., \& Linville, D. (2012). Outness and relationship satisfaction in samegender couples. Journal of Marital and Family Therapy, 38(2), 330-339.

Kwon, P. (2013). Resilience in lesbian, gay, and bisexual individuals. Personality and Social Psychology, 1-13.

Lazarus, R. S., \& Folkman, S. (1984). Stress, appraisal, and coping. New York: Springer.

Legate, N., Ryan, R. M., \& Weinstein, N. (2012). Is coming out always a "good thing"? Exploring the relations of autonomy support, outness, and wellness, for lesbian, gay, and bisexual individuals. Social Psychological and Personality Science, 3(2), $145-152$.

Luthar, S. S., Cicchetti, D., \& Becker, B. (2000). The construct of resilience: A critical evaluation and guidelines for future work. Child Development, 71(3), 543-562.

McCarn, S. R., \& Fassinger, R. E. (1996). Re-visioning sexual minority identity formation: A new model of lesbian identity and its implications for counseling and research. The Counseling Psychologist, 24(3), 508-534.

Menard, S. (Ed.). (2002). Applied logistic regression analysis (Vol. 106). Sage.

Meyer, I. H. (2007). Prejudice and discrimination as social stressors. The health of sexual minorities, Part III, 242-267. 
Meyer, I.H. (2010). Identity, stress, and resilience in lesbians, gay men, and bisexuals of color. The Counseling Psychologist, 38(3), 442-454.

Meyer, I.H. (2013). Prejudice, social stress, and mental health in lesbian, gay, and bisexual populations: Conceptual issues and research evidence. Psychology of Sexual Orientation and Gender Diversity, 1(S), 3-26.

Miller,D. B., \& MacIntosh, R. (1999). Promoting resilience in urban African American adolescents: Racial socialization and identity as protective factors. Social Work Research, 23(3), 159-169.

Mohr, J. J., \& Kendra, M. S. (2011). Revision and extension of a multidimensional measure of sexual minority identity: The lesbian, gay, and bisexual identity scale. Journal of Counseling Psychology, 58(2), 234-245.

Mohr, J., \& Fassinger, R. (2000). Measuring dimensions of lesbian and gay male experience. Measurement and Evaluation in Counseling and Development, 33, $66-90$.

Morris, J. F., Waldo, C. R., \& Rothblum, E. D. (2001). A model of predictors and outcomes of outness among lesbian and bisexual women. American Journal of Orthopsychiatry, 71(1), 61-71.

Murdock, T. B., \& Bolch, M. B. (2005). Risk and protective factors for poor school adjustment in lesbian, gay, and bisexual (LGB) high school youth: Variable and person-centered analyses. Psychology in the School, 42(2), 159-172.

Neville, S. \& Henrickson, M. (2005). Perceptions of lesbian, gay, and bisexual people of primary healthcare services. Issues and Innovations in Nursing and Practice, $55(4), 407-415$. 
Perez, R. M., DeBord, K. A., \& Bieschke, K. J. (2000). Handbook of counseling and psychotherapy with lesbian, gay, and bisexual clients (pp. 35-56). Washington, DC: American Psychological Association.

Reynolds, A. L., \& Hanjorgiris, W. F. (2000). Coming out: Lesbian, gay, and bisexual identity development. In R. M. Perez, K. A. DeBord, \& K. J. Bieschke (Eds.), Handbook of counseling and psychotherapy with lesbian, gay, and bisexual clients (pp. 35-56). Washington, DC: American Psychological Association.

Riggle, E. D. B., Whitman, J. S., Olson, A., Rostosky, S. S., Strong, S. (2008). The positive aspects of being a lesbian or gay man. Professional Psychology: Research and Practice, 39(2), 210-217.

Rosario, M., Schrimshaw, E. W., \& Hunter, J. (2004). Ethnic/racial differences in the coming-out process of lesbian, gay, and bisexual youths: A comparison of sexual identity development over time. Cultural Diversity and Ethnic Minority Psychology, 10(3), 215.

Rothblum, E. D. (2000). " Somewhere in Des Moines or San Antonio": Historical perspectives on lesbian, gay, and bisexual mental health. In R. M. Perez, K. A. DeBord, \& K. J. Bieschke (Eds.), Handbook of counseling and psychotherapy with lesbian, gay, and bisexual clients (pp. 57-79). Washington, DC: American Psychological Association.

Russell, G. M., \& Richards, J. A. (2003). Stressor and resilience factors for Lesbians, gay men, and bisexuals confronting antigay politics. American Journal of Community Psychology, 31(3/4), 313-328.

Rosario, M., Schrimshaw, E. W., Hunter, J., \& Braun, L. (2006). Sexual identity 
development among lesbian, gay, and bisexual youths: Consistency and change over time. Journal of Sex Research, 43(1), 46-58.

Rostosky, S. S., \& Riggle, E. D. (2002). " Out" at work: The relation of actor and partner workplace policy and internalized homophobia to disclosure status. Journal of Counseling Psychology, 49(4), 411.

Savin-Williams, R. C. (2001). A critique of research on sexual-minority youths. Journal of Adolescence, 24, 5-13.

Saewyc, E. M. (2011). Research on adolescent sexual orientation: Development, health disparities, stigma, and resilience. Journal of Research on Adolescence, 21(1), 256-272.

Scourfield, J. Roen, K., \& McDermott, L. (2008). Lesbian, gay, bisexual and transgender young people's experiences of distress: Resilience, ambivalence and selfdestructive behaviour. Health and Social Care in the Community, 16(3), 329-336.

Smith, M., \& Gray, S. W. (2009). The courage to challenge: A new measure of hardiness in LGBT adults. Journal of Gay \& Lesbian Social Services, 21, 73-89.

Sophie, J. (1986). A critical examination of stage theories of lesbian identity development. Journal of Homosexuality, 12(2), 39-51.

Sue, D. W., Bingham, R. P., Porche-Burke, L., Vasquez, M. (1999). The diversification of psychology: A multicultural revolution. The American Psychologist, 54(12), 1061-1069.

Sue, D. W., Capodilupo, C. M., Torino, G. C., Bucceri, J. M., Holder, A., Nadal, K. L., \& Esquilin, M. (2007). Racial microaggressions in everyday life: implications for clinical practice. American Psychologist, 62(4), 271-286 
Swim, J. K., Johnston, K., \& Pearson, N. B. (2009). Daily experiences with heterosexism: Relations between heterosexist hassles and psychological wellbeing. Journal of Social and Clinical Psychology, 28(5), 597-629.

Tabachnick, B. G., and Fidell, L. S. (2006). Using Multivariate Statistics_(5th ed.) Boston : Allyn and Bacon.

Troido, R. R. (1988). Homosexual identity development. Journal of Adolescent Health Care, 9, 105-113.

Ungar, M. (2010). Cultural dimensions of resilience among adults. In J. W. Reich, A. J. Zautra, \& J. S. Hall (Eds.), Handbook of Adult Resilience (404-426). New York, NY: The Guilford Press.

University of Chicago. The Social Organization of Sexuality, Chicago, IL, 1994.

Vaughan, M. D., \& Waehler, C. A. (2010). Coming out growth: Conceptualizing and measuring stress-related growth associated with coming out to others as a sexual minority. Journal of Adult Development, 17(2), 94-109.

Waldo, C. R. (1999). Working in a majority context: A structural model of heterosexism as minority stress in the workplace. Journal of Counseling Psychology, 46(2), 218-232.

Walker, J. N. J., \& Longmire-Avital, B. (2013). The impact of religious faith and internalized homonegativity on resiliency for black lesbian, gay, and bisexual emerging adults. Developmental psychology, 49(9), 1723-1731

White, D., \& Stephenson, R. (2014). Identity Formation, Outness, and Sexual Risk Among Gay and Bisexual Men. American journal of men's health, 8(2), 98-109.

Worthington, R. L., \& Reynolds, A. L. (2009). Within-group differences in sexual 
orientation and identity. Journal of Counseling Psychology, 56(1), 44-55.

Zautra, A. J., Hall, J. S., \& Murray, K. E. (2010). Resilience: A new definition of health for people and communities. In J. W. Reich, A. J. Zautra, \& J. S. Hall (Eds.), Handbook of Adult Resilience (3-34). New York, NY: The Guilford Press.

Zimmerman, M. A., \& Brenner, A. B. (2010). Resilience in adolescence: Overcoming neighborhood disadvantage. In J. W. Reich, A. J. Zautra, \& J. S. Hall (Eds.), Handbook of Adult Resilience (283-308). New York, NY: The Guilford Press. 
Table 1

Participant Demographics

\begin{tabular}{|c|c|c|}
\hline Category & $n$ & $\%$ \\
\hline \multicolumn{3}{|l|}{ Sexual Orientation } \\
\hline Lesbian & 92 & 36.8 \\
\hline Gay & 106 & 42.4 \\
\hline Bisexual & 52 & 20.8 \\
\hline \multicolumn{3}{|l|}{ Gender } \\
\hline Female & 138 & 55.2 \\
\hline Male & 105 & 42 \\
\hline Transgender & 3 & 1.2 \\
\hline Other & 4 & 1.6 \\
\hline \multicolumn{3}{|l|}{$\operatorname{Age}^{a}$} \\
\hline $18-22$ & 55 & 22 \\
\hline $23-25$ & 31 & 12.4 \\
\hline $26-29$ & 27 & 10.8 \\
\hline $30-35$ & 44 & 17.6 \\
\hline $36-39$ & 18 & 7.2 \\
\hline $40-45$ & 15 & 6 \\
\hline $46-49$ & 16 & 6.4 \\
\hline $50-55$ & 20 & 8 \\
\hline $56-59$ & 9 & 3.6 \\
\hline $60-65$ & 8 & 3.2 \\
\hline$\geq 66$ & 7 & 2.8 \\
\hline \multicolumn{3}{|l|}{ Race/Ethnicity } \\
\hline White & 202 & 80.8 \\
\hline Latino/a & 19 & 7.6 \\
\hline African American & 9 & 3.6 \\
\hline American Indian & 2 & 0.8 \\
\hline Chinese & 2 & 0.8 \\
\hline Korean & 2 & 0.8 \\
\hline Asian Indian & 3 & 1.2 \\
\hline Other Asian & 3 & 1.2 \\
\hline Other & 5 & 2 \\
\hline Not Specified & 3 & 1.2 \\
\hline \multicolumn{3}{|c|}{ Religious/Spiritual Orientation } \\
\hline Christian & 72 & 28.8 \\
\hline Jewish & 15 & 6 \\
\hline Unitarian/Universalist & 7 & 2.8 \\
\hline Buddhist & 3 & 1.2 \\
\hline Hindu & 1 & 0.4 \\
\hline Muslim & 1 & 0.4 \\
\hline Native American & 2 & 0.8 \\
\hline Agnostic & 40 & 16 \\
\hline Atheist & 38 & 15.2 \\
\hline
\end{tabular}




\begin{tabular}{lll} 
No Religion & 41 & 16.4 \\
Other & 18 & 7.2 \\
$\quad$ Not Specified & 11 & 4.4 \\
Relationship Status & & \\
$\quad$ Single & 78 & 31.2 \\
Dating & 23 & 9.2 \\
Monogamously Coupled & 78 & 31.2 \\
Civilly Joined/Married & 58 & 23.2 \\
Separated/Divorced & 4 & 1.6 \\
Widowed & 2 & 0.8 \\
Other & 6 & 2.4 \\
Education & & \\
$\quad \leq$ High School Graduate & 5 & 2 \\
Some College & 50 & 20 \\
Associates Degree & 6 & 2.4 \\
Bachelors Degree & 62 & 24.8 \\
Masters Degree & 70 & 28 \\
Doctorate Degree & 55 & 22 \\
\hline${ }^{a}$ M $\approx$ Md $\approx 4$ & &
\end{tabular}

${ }^{\mathrm{a}} \mathrm{M} \approx 34 ; \mathrm{Md} \approx 42$ 
Table 2

Descriptive Statistics for All Instruments and Subscales

\begin{tabular}{llllll}
\hline Instrument & Mean & SD & $\alpha$ & Scale $^{\mathrm{a}}$ & No. Items \\
\hline OI & 5.07 & 1.14 & .75 & $1-7^{\mathrm{b}}$ & 11 \\
CCS & 5.72 & .60 & .86 & $1-7$ & 18 \\
GALOSI-F & 2.30 & .50 & .94 & $1-6$ & 49 \\
GALOSI-E & 3.36 & 1.08 & .94 & $1-6^{\mathrm{b}}$ & 47 \\
LGBIS & & & & $1-6$ & 27 \\
$\quad$ Concealment Motivation & 2.59 & 1.02 & .81 & & 3 \\
$\quad$ Identity Uncertainty & 1.56 & .81 & .84 & & 4 \\
$\quad$ Internalized Homonegativity & 1.66 & .91 & .88 & & 3 \\
$\quad$ Difficult Process & 2.88 & .69 & .80 & & 3 \\
$\quad$ Acceptance Concerns & 2.96 & 1.09 & .79 & & 3 \\
$\quad$ Identity Superiority & 1.87 & .85 & .73 & & 3 \\
$\quad$ Identity Affirmation & 5.14 & .78 & .87 & & 3 \\
$\quad$ Identity Centrality & 4.09 & .72 & .86 & & 5 \\
\hline All scates Likert-type. & & & & & \\
\hline
\end{tabular}

aAll scales Likert-type.
${ }^{\mathrm{b}}$ Scale also included a "Not Applicable" response option. 
Table 3

Pearson Correlations for Outness

\begin{tabular}{llllll}
\hline & Resilience & EPD & FPD & $\mathrm{R} \times$ EPD & $\mathrm{R} \times \mathrm{FPD}$ \\
\hline$r$ & .268 & -.137 & -.260 & .008 & .008 \\
Sig (2-tailed) & $<.001$ & $<.05$ & $<.001$ & $\mathrm{~ns}$ & $\mathrm{~ns}$ \\
\hline
\end{tabular}


Table 4

Hierarchical Regression for Outness

\begin{tabular}{lllll}
\hline Regression Model & $\beta$ & $\mathrm{R}$ & $\mathrm{R}^{2}$ & $\mathrm{~F}$ \\
\hline Model 1 & & $.363^{* * *}$ & .132 & 7.42 \\
Resilience & $.241^{* * *}$ & & & \\
EPD & .087 & & \\
FPD & $-.306^{* *}$ & & \\
R $\times$ EPD & -.044 & & \\
R $\times$ FPD & .036 & & \\
\hline
\end{tabular}

Note. All beta coefficients are standardized.

$* * p<.01 ; * * * p<.001$ for models and predicting variables. 
Table 5

Forward Regression for Outness

\begin{tabular}{lllll} 
Regression Model & $\beta$ & $\mathrm{R}$ & $\mathrm{R}^{2}$ & $\mathrm{~F}$ \\
$\begin{array}{l}\text { Model 1 } \\
\quad \text { Resilience } \\
\begin{array}{l}\text { Model 2 } \\
\text { Resilience }\end{array}\end{array}$ & $.268^{* * *}$ & $.268^{* * *}$ & .072 & 19.18 \\
$\quad$ FPD & $.248^{* * *}$ & $.358^{* * *}$ & .128 & 18.17 \\
\hline
\end{tabular}

Note. All beta coefficients are standardized.

$* * * p<.001$ for models and predicting variables. 
Table 6

Backward Regression for Outness

\begin{tabular}{|c|c|c|c|c|}
\hline Regression Model & $\beta$ & $\mathrm{R}$ & $\mathrm{R}^{2}$ & $\mathrm{~F}$ \\
\hline Model 1 & & $.363 * * *$ & .132 & 7.42 \\
\hline Resilience & $.241 * * *$ & & & \\
\hline EPD & .087 & & & \\
\hline FPD & $-.306 * *$ & & & \\
\hline $\mathrm{R} \times \mathrm{EPD}$ & -.044 & & & \\
\hline $\mathrm{R} \times \mathrm{FPD}$ & .036 & & & \\
\hline Model 2 & & $.362 * * *$ & .131 & 9.25 \\
\hline Resilience & $.242 * * *$ & & & \\
\hline EPD & .077 & & & \\
\hline FPD & $-.297 * *$ & & & \\
\hline $\mathrm{R} \times \mathrm{EPD}$ & -.019 & & & \\
\hline Model 3 & & $.362 * * *$ & .131 & 12.35 \\
\hline Resilience & $.243 * * *$ & & & \\
\hline EPD & .075 & & & \\
\hline FPD & $-.293 * *$ & & & \\
\hline Model 4 & & $.358 * * *$ & .128 & 18.17 \\
\hline Resilience & $.248 * * *$ & & & \\
\hline FPD & $-.239 * * *$ & & & \\
\hline
\end{tabular}

Note. All beta coefficients are standardized.

$* * p<.01 ; * * * p<.001$ for models and predicting variables. 
Table 7

Pearson Correlations Between Predictor Variables and LGBIS Subscales

\begin{tabular}{lllllllll}
\hline \multicolumn{2}{c}{$\mathrm{CM}$} & $\mathrm{IU}$ & $\mathrm{AC}$ & $\mathrm{IA}$ & $\mathrm{IH}$ & $\mathrm{DP}$ & $\mathrm{IS}$ & $\mathrm{IC}$ \\
\hline $\mathrm{Res}$ & $-.248^{* * *}$ & $-.205^{* *}$ & $-.318^{* * *}$ & $.345^{* * *}$ & $-.283^{* * *}$ & $-.212^{* *}$ & $-.206^{* *}$ & .059 \\
$\mathrm{EPD}$ & $.130^{*}$ & $.139^{*}$ & $.224^{* * *}$ & -.013 & .054 & $.213^{* *}$ & .096 & .121 \\
$\mathrm{FPD}$ & $.277^{* * *}$ & .064 & $.478^{* * *}$ & $-.156^{*}$ & $.259^{* * *}$ & $.430 * * *$ & .096 & .082 \\
\hline
\end{tabular}

Note. $\mathrm{CM}=$ Concealment Motivation; IU = Identity Uncertainty; AC = Acceptance

Concerns; IA = Identity Affirmation; IH = Internalized Homonegativity; DP = Difficult

Process; IS = Identity Superiority; IC = Identity Centrality; Res = Resilience; EPD = effect of perceived discrimination; and $\mathrm{FPD}=$ frequency of perceived discrimination. $* p<.05 ; * * p<.01 ; * * * p<.001$ 
Table 8

Descriptive Statistics and Pearson Correlations for Outness by Demographics

\begin{tabular}{|c|c|c|c|c|c|c|c|}
\hline \multirow[b]{2}{*}{ Category } & \multicolumn{3}{|c|}{ Resilience } & \multicolumn{3}{|c|}{ FPD } & \multirow[b]{2}{*}{$n$} \\
\hline & $r$ & $M$ & $S D$ & $r$ & $M$ & $S D$ & \\
\hline \multicolumn{8}{|l|}{ Sexual Orientation } \\
\hline Lesbian & $.290 * *$ & 5.79 & .60 & $-.381 * * *$ & 2.23 & .49 & 92 \\
\hline Gay & $.276 * *$ & 5.70 & .58 & $-.234 * *$ & 2.36 & .49 & 106 \\
\hline Bisexual & .189 & 5.65 & .63 & -.180 & 2.27 & .52 & 52 \\
\hline \multicolumn{8}{|l|}{ Gender } \\
\hline Female & $.259 * *$ & 5.77 & .61 & $-.326 * * *$ & 2.24 & .49 & 138 \\
\hline Male & $.248 *$ & 5.66 & .58 & -.138 & 2.37 & .48 & 105 \\
\hline \multicolumn{8}{|l|}{ Age } \\
\hline $18-29$ & $.268 * *$ & 5.62 & .62 & $-.174^{*}$ & 2.36 & .55 & 113 \\
\hline $30-45$ & .094 & 5.78 & .60 & $-.268 * *$ & 2.29 & .44 & 77 \\
\hline $46-66+$ & $.297 *$ & 5.85 & .51 & $-.359 * *$ & 2.19 & .44 & 60 \\
\hline \multicolumn{8}{|l|}{ Race/Ethnicity } \\
\hline White & $.238 * * *$ & 5.72 & .59 & $-.286 * * *$ & 2.28 & .49 & 202 \\
\hline Non-White & $.409 * *$ & 5.72 & .63 & -.161 & 2.33 & .50 & 48 \\
\hline \multicolumn{8}{|l|}{ Religion } \\
\hline Religious & $.268 * *$ & 5.72 & .64 & $-.354 * * *$ & 2.37 & .53 & 101 \\
\hline Non-Religious & $.323 * * *$ & 5.74 & .57 & $-.171 *$ & 2.21 & .47 & 119 \\
\hline \multicolumn{8}{|l|}{ Relationship } \\
\hline Partner & $.243 * *$ & 5.74 & .61 & $-.303 * * *$ & 2.28 & .48 & 159 \\
\hline No Partner & $.275 * *$ & 5.65 & .59 & -.123 & 2.35 & .52 & 84 \\
\hline \multicolumn{8}{|l|}{ Education } \\
\hline$\leq$ Some College & .113 & 5.64 & .59 & $-.217^{*}$ & 2.39 & .58 & 61 \\
\hline Bachelors Level & $.466 * * *$ & 5.77 & .62 & $-.219^{*}$ & 2.32 & .49 & 62 \\
\hline$\geq$ Masters Level & $.233 * *$ & 5.73 & .60 & $-.267 * *$ & 2.24 & .45 & 125 \\
\hline
\end{tabular}


Table 9

Multiple Regressions for Outness by Demographics

\begin{tabular}{|c|c|c|c|c|c|}
\hline \multirow[b]{2}{*}{ Category } & \multicolumn{2}{|c|}{$\beta$} & \multirow[b]{2}{*}{$\mathrm{R}$} & \multirow[b]{2}{*}{$\mathrm{R}^{2}$} & \multirow[b]{2}{*}{$\mathrm{F}$} \\
\hline & Res & FPD & & & \\
\hline \multicolumn{6}{|l|}{ Sexual Orientation } \\
\hline Lesbian & $.230 *$ & $-.341 * *$ & $.447 * * *$ & .200 & 11.11 \\
\hline Gay & $.239 * *$ & -.195 & $.347 * *$ & .121 & 7.07 \\
\hline Bisexual & .200 & -.189 & .267 & .072 & 1.89 \\
\hline \multicolumn{6}{|l|}{ Gender } \\
\hline Female & $.241 * *$ & $-.327 * * *$ & $.399 * * *$ & .159 & 12.79 \\
\hline Male & $.220 *$ & -.116 & $.278 *$ & .077 & 4.27 \\
\hline \multicolumn{6}{|l|}{ Age } \\
\hline $18-29$ & $.234 * *$ & -.129 & $.306 * *$ & .094 & 5.67 \\
\hline $30-45$ & .099 & $-.278 *$ & $.290 *$ & .084 & 3.39 \\
\hline $46-66+$ & $.200 *$ & $-.251 *$ & $.433 * *$ & .187 & 6.56 \\
\hline \multicolumn{6}{|l|}{ Race/Ethnicity } \\
\hline White & $.217 * *$ & $-.262 * * *$ & $.367 * * *$ & .134 & 15.45 \\
\hline Non-White & $.416^{* *}$ & -.045 & $.410 *$ & .168 & 4.56 \\
\hline \multicolumn{6}{|l|}{ Religion } \\
\hline Religious & $.216^{*}$ & $-.315 * *$ & $.416 * * *$ & .173 & 10.25 \\
\hline Non-Religious & $.324 * * *$ & $-.160 *$ & $.357 * * *$ & .128 & 8.48 \\
\hline \multicolumn{6}{|l|}{ Relationship Status } \\
\hline Partnered & $.217 * *$ & $-.284 * * *$ & $.384 * * *$ & .147 & 13.47 \\
\hline Non-Partnered & $.274 *$ & -.080 & $.287 *$ & .082 & 3.64 \\
\hline \multicolumn{6}{|l|}{ Education } \\
\hline$\leq$ Some College & .113 & $-.179 *$ & .245 & .060 & 1.86 \\
\hline Bachelors Level & $.492 * * *$ & -.115 & $.476 * *$ & .226 & 8.63 \\
\hline$\geq$ Masters Level & $.178 * *$ & $-.229 * *$ & $.348 * * *$ & .121 & 8.41 \\
\hline
\end{tabular}

Note. All beta coefficients are standardized.

${ }^{*} p<.05 ;{ }^{*} p<.01$; and ${ }^{* *} p<.001$ for all models and predicting variables. 
Figure 1

Proposed Role of Environmental Factors in Identity Integration.

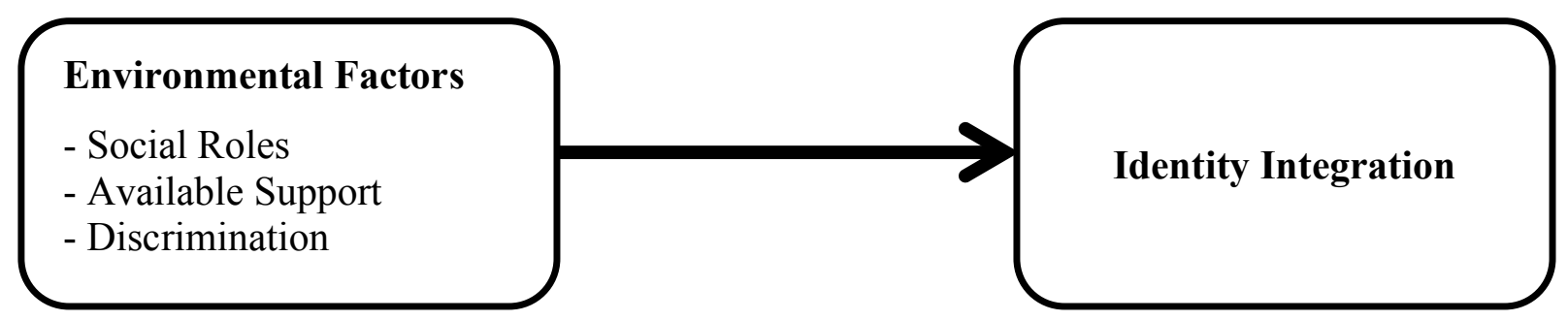


Figure 2

Proposed Moderating Effect of Resilience.

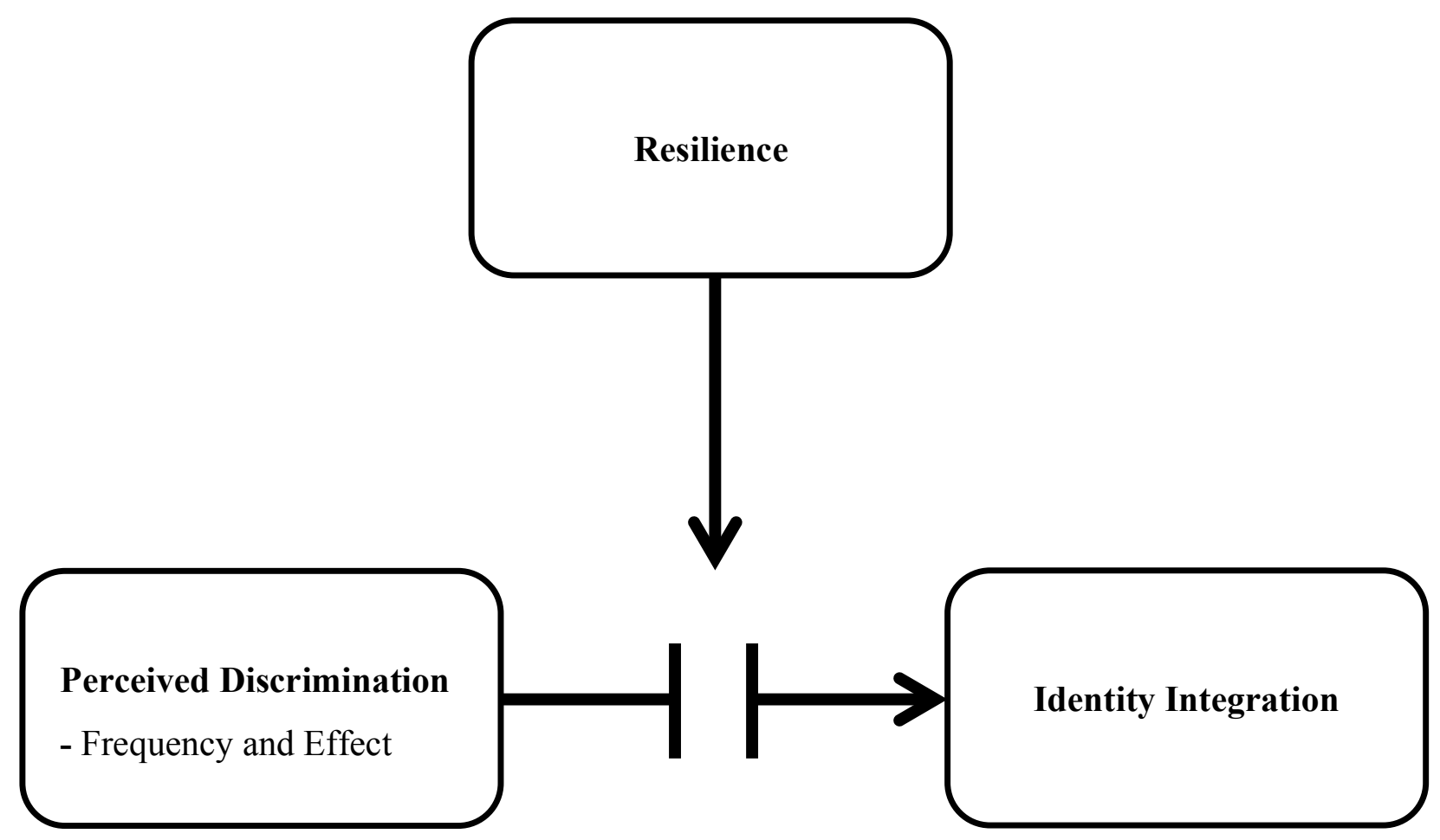




\section{APPENDIX A}

\section{Demographic Questionnaire}

Please answer the following questions about yourself. Feel free to select 'Not specified' for any question that you are unable to answer, but please do not leave anything blank. Once you've finished, please click the arrows at the bottom of this screen to begin the survey. Thank you for participating. [Note to reader: With the exception of items 8 and 9, the following category selections appeared as drop-down boxes in the online format of the final survey. Respondents were able to select only one option for each category.]

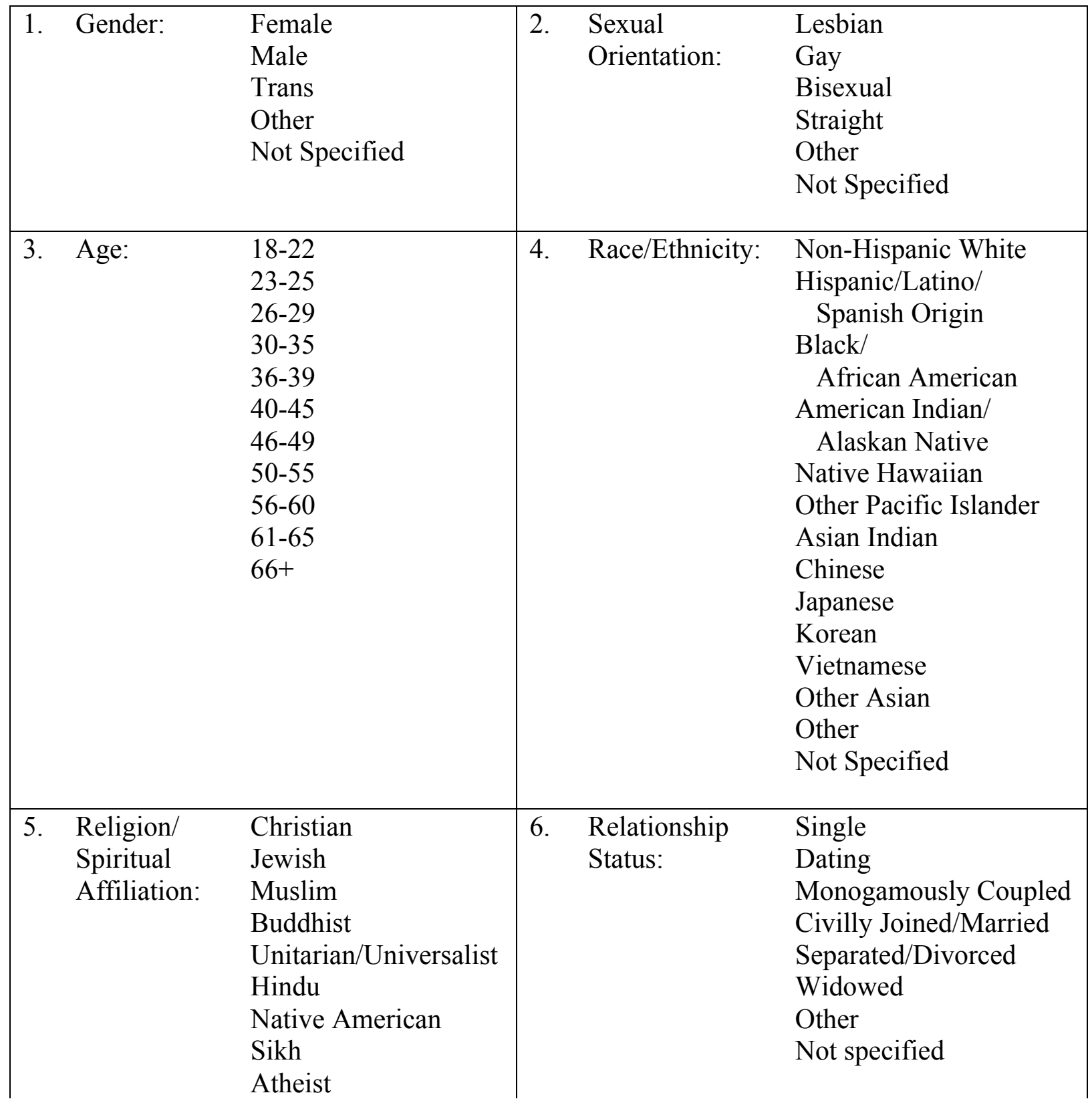




\begin{tabular}{|c|c|c|}
\hline & $\begin{array}{l}\text { Agnostic } \\
\text { No Religion } \\
\text { Other } \\
\text { Not Specified }\end{array}$ & \\
\hline 7. Education: & $\begin{array}{l}\text { Some High School } \\
\text { High School Graduate } \\
\text { Some College } \\
\text { Associates Degree } \\
\text { Bachelors Degree } \\
\text { Masters Degree } \\
\text { Doctoral Degree } \\
\text { Other } \\
\text { Not Specified }\end{array}$ & \\
\hline 8. Occupation: & & $\begin{array}{ll}\text { 9. } & \text { State of } \\
& \text { Residence: }\end{array}$ \\
\hline
\end{tabular}




\section{APPENDIX B}

\section{Outness Inventory}

Use the following rating scale to indicate how open you are about your sexual orientation to the people listed below. Try to respond to all of the items, but leave items blank if they do not apply to you.

$1=$ Person definitely does NOT know about your sexual orientation status

$2=$ Person might know about your sexual orientation status, but it is NEVER talked about

3 = Person probably knows about your sexual orientation status, but it is NEVER talked about

4 = Person probably knows about your sexual orientation status, but it is RARELY talked about

5 = Person definitely knows about your sexual orientation status, but it is RARELY talked about

6 = Person definitely knows about your sexual orientation status, and it is SOMETIMES talked about

7 = Person definitely knows about your sexual orientation status, and it is OPENLY talked about

$0=$ Not applicable to your situation; there is no such person or group of people in your life

\begin{tabular}{|llllllllll|}
\hline 1. & Mother & 1 & 2 & 3 & 4 & 5 & 6 & 7 & 0 \\
\hline 2. & Father & 1 & 2 & 3 & 4 & 5 & 6 & 7 & 0 \\
\hline 3. & Siblings (sisters, brothers) & 1 & 2 & 3 & 4 & 5 & 6 & 7 & 0 \\
\hline 4. & Extended family/relatives & 1 & 2 & 3 & 4 & 5 & 6 & 7 & 0 \\
\hline 5. & My new straight friends & 1 & 2 & 3 & 4 & 5 & 6 & 7 & 0 \\
\hline 6. & My work peers & 1 & 2 & 3 & 4 & 5 & 6 & 7 & 0 \\
\hline 7. & My work supervisor(s) & 1 & 2 & 3 & 4 & 5 & 6 & 7 & 0 \\
\hline 8. & $\begin{array}{l}\text { Members of my religious community (e.g., } \\
\text { church, temple) }\end{array}$ & 1 & 2 & 3 & 4 & 5 & 6 & 7 & 0 \\
\hline 9. & $\begin{array}{l}\text { Leaders of my religious community (e.g., } \\
\text { church, temple) }\end{array}$ & 1 & 2 & 3 & 4 & 5 & 6 & 7 & 0 \\
\hline 10. & Strangers, new acquaintances & 1 & 2 & 3 & 4 & 5 & 6 & 7 & 0 \\
\hline 11. & My old heterosexual friends & 1 & 2 & 3 & 4 & 5 & 6 & 7 & 0 \\
\hline
\end{tabular}




\section{APPENDIX C}

\section{Courage to Challenge Scale}

Please answer by marking how accurately the following statements match your own feelings.

$1=$ Strongly Disagree

$2=$ Disagree

$3=$ Mildly Disagree

$4=$ Neutral

$5=$ Mildly Agree

$6=$ Agree

$7=$ Strongly Agree

1. I believe that things usually turn out for the best.

$\begin{array}{lllllll}1 & 2 & 3 & 4 & 5 & 6 & 7\end{array}$

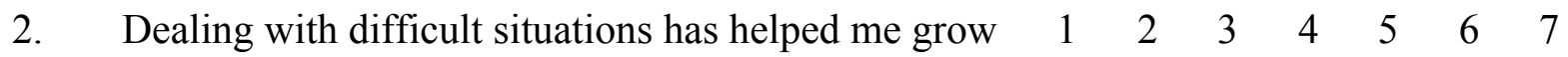
in positive ways.

3. When I encounter people's hostile attitudes, I can $\quad \begin{array}{lllllll}1 & 2 & 3 & 4 & 5 & 6 & 7\end{array}$ control my reactions.

4. When people don't support me, it doesn't stop me $\quad \begin{array}{llllllll}1 & 2 & 3 & 4 & 5 & 6 & 7\end{array}$ from going ahead with my goals.

5. I guess I'm pretty tough because I've gotten through $\quad \begin{array}{llllllll}1 & 2 & 3 & 4 & 5 & 6 & 7\end{array}$ some hard times.

6. I don't let fear rule my life.

$\begin{array}{lllllll}1 & 2 & 3 & 4 & 5 & 6 & 7\end{array}$

7. Believing in myself helps me get through hard times. $\begin{array}{llllllll}1 & 2 & 3 & 4 & 5 & 6 & 7\end{array}$

8. I'm determined to reach my goals in life. $\quad \begin{array}{llllllll}1 & 2 & 3 & 4 & 5 & 6 & 7\end{array}$

9. I'm convinced that if you put your mind to it, you $\quad \begin{array}{llllllll}1 & 2 & 3 & 4 & 5 & 6 & 7\end{array}$ can do almost anything.

10. I have the courage to stand up for what's right.

$\begin{array}{lllllll}1 & 2 & 3 & 4 & 5 & 6 & 7\end{array}$

11. It's important to me to be honest about who I am. $\quad \begin{array}{llllllll}1 & 2 & 3 & 4 & 5 & 6 & 7\end{array}$

12. When people don't support me, it doesn't get me $\quad \begin{array}{llllllll}1 & 2 & 3 & 4 & 5 & 6 & 7\end{array}$ down.

13. Getting through tough times prepares me for future $\begin{array}{llllllll}1 & 2 & 3 & 4 & 5 & 6 & 7\end{array}$ 
challenges.

14. My sense of humor helps get me through tough $\quad \begin{array}{lllllllll}1 & 2 & 3 & 4 & 5 & 6 & 7\end{array}$ times.

15. Integrity is an important personal value of mine. $\quad \begin{array}{llllllll}1 & 2 & 3 & 4 & 5 & 6 & 7\end{array}$

16. Even in the midst of very stressful times, I can find $\begin{array}{llllllll}1 & 2 & 3 & 4 & 5 & 6 & 7\end{array}$ something to laugh about.

17. I guess I have spirit ... it's hard to keep me down. $\quad \begin{array}{llllllll}1 & 2 & 3 & 4 & 5 & 6 & 7\end{array}$

18. Finding courage to come out has made me a much $\quad \begin{array}{lllllllll}1 & 2 & 3 & 4 & 5 & 6 & 7\end{array}$ better person. 


\section{APPENDIX D}

\section{Gay and Lesbian Oppressive Situations Inventory}

Gay men, lesbians, and bisexuals often encounter discrimination, prejudice, and negative stereotypes based on their sexual orientation. Below are situations that you may have encountered. Please think about each situation and how often you have experienced it. Also consider the effect or impact each situation has had on you. Use the following scales when responding to these situations. Please answer all questions.

\begin{tabular}{|c|c|c|c|c|c|c|c|c|c|c|c|}
\hline & \multicolumn{5}{|c|}{ Frequency } & \multicolumn{6}{|c|}{ Effect } \\
\hline I have had anti-LGB remarks directed at me. & 1 & 2 & 3 & 4 & 5 & 1 & 2 & 3 & 4 & 5 & $\mathrm{~N} / \mathrm{A}$ \\
\hline $\begin{array}{l}\text { I have been uncomfortable about introducing } \\
\text { my partner/girlfriend/boyfriend to biological } \\
\text { family members. }\end{array}$ & 1 & 2 & 3 & 4 & 5 & 1 & 2 & 3 & 4 & 5 & $\mathrm{~N} / \mathrm{A}$ \\
\hline $\begin{array}{l}\text { People have avoided me because of my sexual } \\
\text { orientation. }\end{array}$ & 1 & 2 & 3 & 4 & 5 & 1 & 2 & 3 & 4 & 5 & $\mathrm{~N} / \mathrm{A}$ \\
\hline $\begin{array}{l}\text { I have seen the media negatively portray gays, } \\
\text { lesbians, and bisexuals. }\end{array}$ & 1 & 2 & 3 & 4 & 5 & 1 & 2 & 3 & 4 & 5 & $\mathrm{~N} / \mathrm{A}$ \\
\hline I have heard people tell gay-bashing jokes. & 1 & 2 & 3 & 4 & 5 & 1 & 2 & 3 & 4 & 5 & $\mathrm{~N} / \mathrm{A}$ \\
\hline $\begin{array}{l}\text { Advancement opportunities at work have } \\
\text { been limited because of my sexual } \\
\text { orientation. }\end{array}$ & 1 & 2 & 3 & 4 & 5 & 1 & 2 & 3 & 4 & 5 & $\mathrm{~N} / \mathrm{A}$ \\
\hline $\begin{array}{l}\text { Members of my biological family have made } \\
\text { anti-LGB remarks. }\end{array}$ & 1 & 2 & 3 & 4 & 5 & 1 & 2 & 3 & 4 & 5 & $\mathrm{~N} / \mathrm{A}$ \\
\hline $\begin{array}{l}\text { I have seen people assume that gay men are } \\
\text { HIV+. }\end{array}$ & 1 & 2 & 3 & 4 & 5 & 1 & 2 & 3 & 4 & 5 & $\mathrm{~N} / \mathrm{A}$ \\
\hline $\begin{array}{l}\text { I have felt isolated by members of my } \\
\text { biological family because of my sexual } \\
\text { orientation. }\end{array}$ & 1 & 2 & 3 & 4 & 5 & 1 & 2 & 3 & 4 & 5 & $\mathrm{~N} / \mathrm{A}$ \\
\hline $\begin{array}{l}\text { I have seen people assume that gay men } \\
\text { exhibit indecent and flamboyant behavior. }\end{array}$ & 1 & 2 & 3 & 4 & 5 & 1 & 2 & 3 & 4 & 5 & $\mathrm{~N} / \mathrm{A}$ \\
\hline People have stared at me because I look gay. & 1 & 2 & 3 & 4 & 5 & 1 & 2 & 3 & 4 & 5 & $\mathrm{~N} / \mathrm{A}$ \\
\hline $\begin{array}{l}\text { People have treated me differently if they } \\
\text { think I am LGB. }\end{array}$ & 1 & 2 & 3 & 4 & 5 & 1 & 2 & 3 & 4 & 5 & $\mathrm{~N} / \mathrm{A}$ \\
\hline
\end{tabular}


People have told me to keep my sexual orientation a secret.

I have been physically threatened because of my sexual orientation.

I have been afraid that my family would reject me because of my sexual orientation.

I have been the butt of anti-LGB jokes.

When I was growing up my religion preached that homosexuality is wrong.

I have seen people assume that lesbians are overly masculine women.

I have seen anti-LGB graffiti in public places.

My biological family has denied the existence of LGB family members.

When I was growing up, my religion preached gayness is wrong.

Biological family members have rejected me because of my sexual orientation.

My sexual orientation has been in conflict with my religious beliefs.

I have had to hide my sexual orientation to be accepted by members of my family.

I have known heterosexuals who think that gays are child molesters.

I have had biological family members as me to pretend that I am not LGB.

I have heard people making negative remarks about LGB people.

It has been hard for me to feel good about myself because of people's negative views

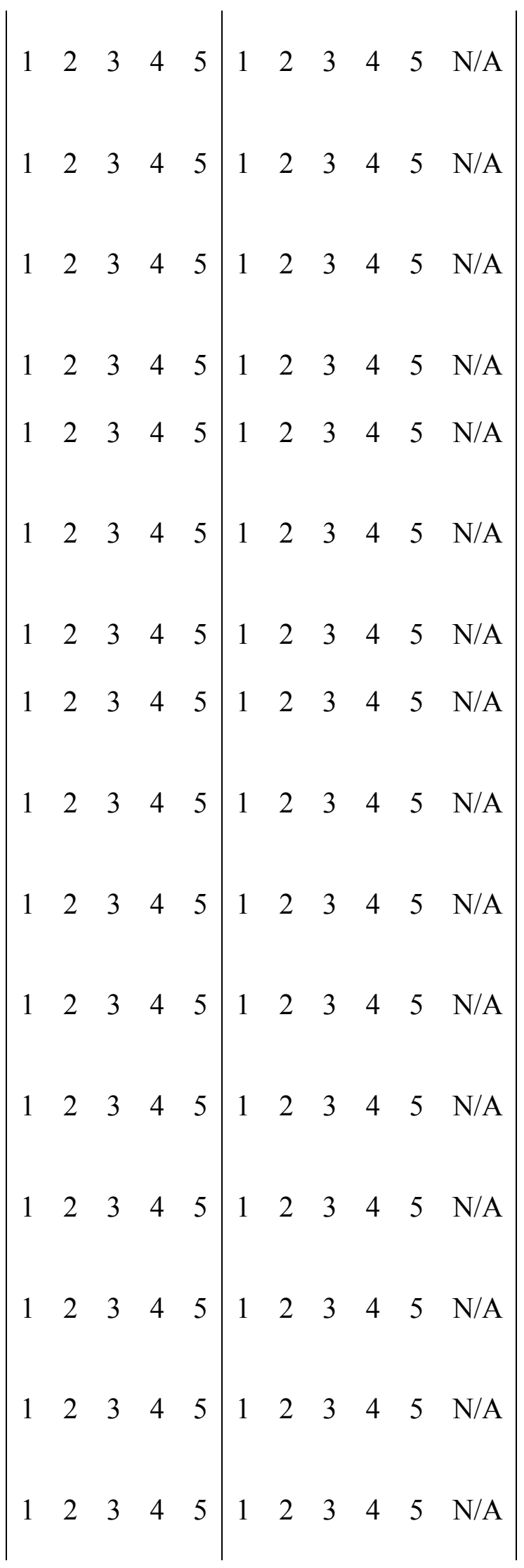




\begin{tabular}{|c|c|c|c|c|c|c|c|c|c|c|c|}
\hline I have known LGB people committed suicide. & 1 & 2 & 3 & 4 & 5 & 1 & 2 & 3 & 4 & 5 & $\mathrm{~N} / \mathrm{A}$ \\
\hline $\begin{array}{l}\text { I have seen that it is harder for LGB people to } \\
\text { have children than it is for heterosexuals. }\end{array}$ & 1 & 2 & 3 & 4 & 5 & 1 & 2 & 3 & 4 & 5 & $\mathrm{~N} / \mathrm{A}$ \\
\hline $\begin{array}{l}\text { It has been hard for me to accept my sexual } \\
\text { orientation. }\end{array}$ & 1 & 2 & 3 & 4 & 5 & 1 & 2 & 3 & 4 & 5 & $\mathrm{~N} / \mathrm{A}$ \\
\hline $\begin{array}{l}\text { It has been hard for me to accept my sexual } \\
\text { orientation. }\end{array}$ & 1 & 2 & 3 & 4 & 5 & 1 & 2 & 3 & 4 & 5 & $\mathrm{~N} / \mathrm{A}$ \\
\hline $\begin{array}{l}\text { I have been denied employment because of } \\
\text { my sexual orientation. }\end{array}$ & 1 & 2 & 3 & 4 & 5 & 1 & 2 & 3 & 4 & 5 & $\mathrm{~N} / \mathrm{A}$ \\
\hline I have denied my sexual orientation. & 1 & 2 & 3 & 4 & 5 & 1 & 2 & 3 & 4 & 5 & $\mathrm{~N} / \mathrm{A}$ \\
\hline $\begin{array}{l}\text { I have been uncomfortable about bringing my } \\
\text { partner / girlfriend / boyfriend to work-related } \\
\text { social events. }\end{array}$ & 1 & 2 & 3 & 4 & 5 & 1 & 2 & 3 & 4 & 5 & $\mathrm{~N} / \mathrm{A}$ \\
\hline $\begin{array}{l}\text { I have been denied housing because of my } \\
\text { sexual orientation. }\end{array}$ & 1 & 2 & 3 & 4 & 5 & 1 & 2 & 3 & 4 & 5 & $\mathrm{~N} / \mathrm{A}$ \\
\hline $\begin{array}{l}\text { I have been worried that people would avoid } \\
\text { me because of my sexual orientation. }\end{array}$ & 1 & 2 & 3 & 4 & 5 & 1 & 2 & 3 & 4 & 5 & $\mathrm{~N} / \mathrm{A}$ \\
\hline $\begin{array}{l}\text { I have had to think about how much of my } \\
\text { sexual orientation to share with new people. }\end{array}$ & 1 & 2 & 3 & 4 & 5 & 1 & 2 & 3 & 4 & 5 & $\mathrm{~N} / \mathrm{A}$ \\
\hline $\begin{array}{l}\text { I have been afraid to publicly display } \\
\text { affection for my partner / girlfriend / } \\
\text { boyfriend. }\end{array}$ & 1 & 2 & 3 & 4 & 5 & 1 & 2 & 3 & 4 & 5 & $\mathrm{~N} / \mathrm{A}$ \\
\hline $\begin{array}{l}\text { I have been afraid of being physically injured } \\
\text { because of my sexual orientation. }\end{array}$ & 1 & 2 & 3 & 4 & 5 & 1 & 2 & 3 & 4 & 5 & $\mathrm{~N} / \mathrm{A}$ \\
\hline $\begin{array}{l}\text { I have known LGB people who have } \\
\text { attempted suicide. }\end{array}$ & 1 & 2 & 3 & 4 & 5 & 1 & 2 & 3 & 4 & 5 & $\mathrm{~N} / \mathrm{A}$ \\
\hline $\begin{array}{l}\text { I have been physically injured because of my } \\
\text { sexual orientation. }\end{array}$ & 1 & 2 & 3 & 4 & 5 & 1 & 2 & 3 & 4 & 5 & $\mathrm{~N} / \mathrm{A}$ \\
\hline $\begin{array}{l}\text { have been stereotyped based on my sexual } \\
\text { orientation. }\end{array}$ & 1 & 2 & 3 & 4 & 5 & 1 & 2 & 3 & 4 & 5 & $\mathrm{~N} / \mathrm{A}$ \\
\hline
\end{tabular}


Members of my biological family have acted like my sexual orientation is wrong.

I have felt depressed about my sexual orientation.

I have known people who have been physically injured because of their sexual orientation.

I have gotten the message that homosexuality is undesirable.

I have seen people assume that gay men have AIDS.

Friends have rejected me because of my sexual orientation.

I have seen the media stereotype gays, lesbians, and/or bisexuals.

I have seen people tell lesbians that all they need is a good man.

I have seen people assume that lesbians hate men.

I have worried that I will go to hell because of my sexual orientation.

Members of my biological family have acted like gayness is wrong.

I have worried about disapproval when I have shared my sexual orientation with heterosexuals.

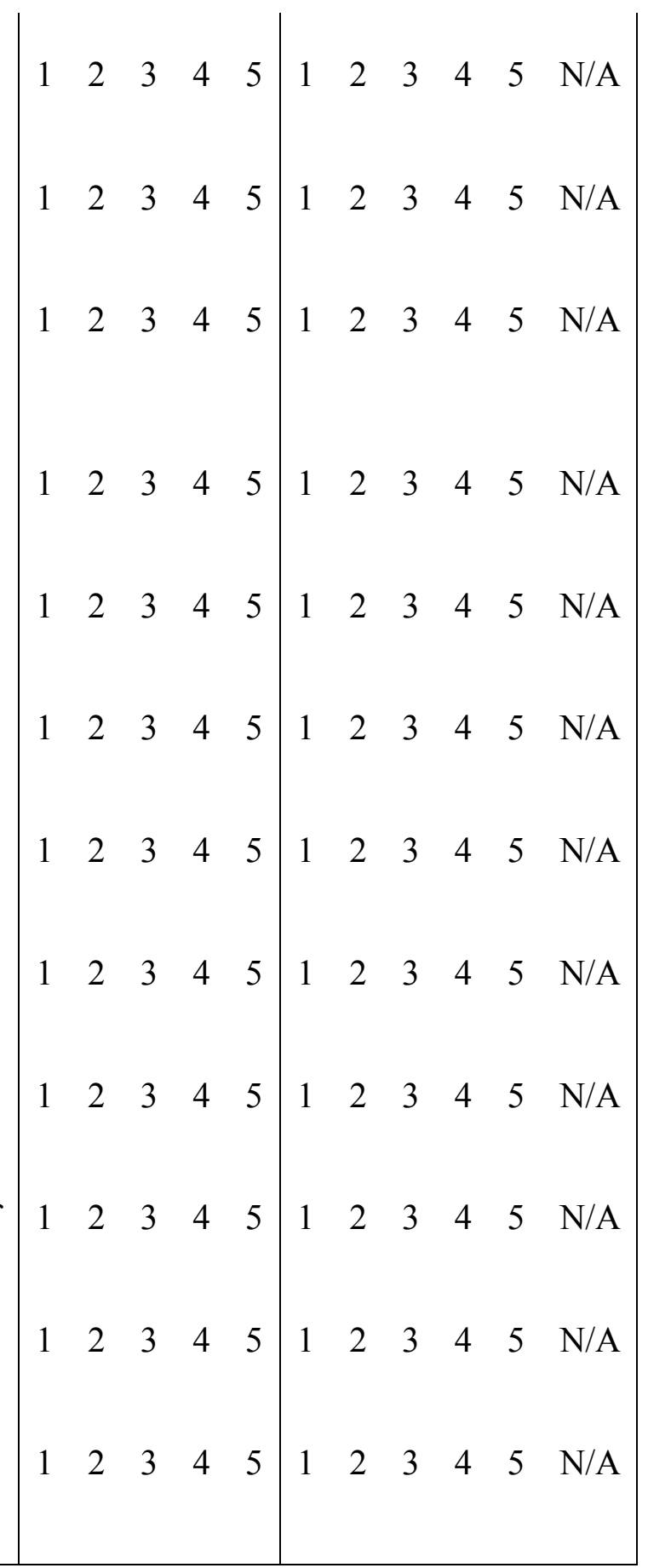




\section{APPENDIX E}

\section{Lesbian, Gay, and Bisexual Identity Scale}

For each of the following questions, please mark the response that best indicates your current experience as an LGB person. Please be as honest as possible: Indicate how you really feel now, not how you think you should feel. There is no need to think too much about any one question. Answer each question according to your initial reaction and then move on to the next.

$1=$ Disagree Strongly

$2=$ Disagree

$3=$ Disagree Somewhat

$4=$ Agree Somewhat

$5=$ Agree

$6=$ Agree Strongly

1. I prefer to keep my same-sex romantic

$\begin{array}{llllll}1 & 2 & 3 & 4 & 5 & 6\end{array}$
relationships rather private.

2. If it were possible, I would choose to be

$\begin{array}{llllll}1 & 2 & 3 & 4 & 5 & 6\end{array}$
straight.

3. I'm not totally sure what my sexual orientation $\quad \begin{array}{lllllll}1 & 2 & 3 & 4 & 5 & 6\end{array}$ is.

$\begin{array}{llllllll}\text { 4. I keep careful control over who knows about my } & 1 & 2 & 3 & 4 & 5 & 6\end{array}$ same-sex romantic relationships.

$\begin{array}{llllllll}\text { 5. I often wonder whether others judge me for my } & 1 & 2 & 3 & 4 & 5 & 6\end{array}$ sexual orientation.

6. I am glad to be an LGB person.

$\begin{array}{llllll}1 & 2 & 3 & 4 & 5 & 6\end{array}$

7. I look down on heterosexuals.

$\begin{array}{llllll}1 & 2 & 3 & 4 & 5 & 6\end{array}$

8. I keep changing my mind about my sexual orientation.

9. I can't feel comfortable knowing that others

$\begin{array}{llllll}1 & 2 & 3 & 4 & 5 & 6\end{array}$
judge me negatively for my sexual orientation.

10. I feel that LGB people are superior to heterosexuals.

$\begin{array}{llllll}1 & 2 & 3 & 4 & 5 & 6\end{array}$

11. My sexual orientation is an insignificant part of $\begin{array}{lllllll}1 & 2 & 3 & 4 & 5 & 6\end{array}$ 
who I am.

12. Admitting to myself that I'm an LGB person has $\begin{array}{lllllll}1 & 2 & 3 & 4 & 5 & 6\end{array}$ been a very painful process.

13. I'm proud to be part of the LGB community. $\quad \begin{array}{lllllll}1 & 2 & 3 & 4 & 5 & 6\end{array}$

14. I can't decide whether I am bisexual or $\quad \begin{array}{lllllll}1 & 2 & 3 & 4 & 5 & 6\end{array}$ homosexual.

15. My sexual orientation is a central part of my $\quad \begin{array}{lllllll}1 & 2 & 3 & 4 & 5 & 6\end{array}$ identity.

16. I think a lot about how my sexual orientation $\quad \begin{array}{llllllll}1 & 2 & 3 & 4 & 5 & 6\end{array}$ affects the way people see me.

17. Admitting to myself that I'm an LGB person has $\begin{array}{lllllll}1 & 2 & 3 & 4 & 5 & 6\end{array}$ been a very slow process.

18. Straight people have boring lives compared with $\quad \begin{array}{lllllll}1 & 2 & 3 & 4 & 5 & 6\end{array}$ LGB people.

19. My sexual orientation is a very personal and $\quad \begin{array}{llllllll}1 & 2 & 3 & 4 & 5 & 6\end{array}$ private matter.

20. I wish I were heterosexual. $\quad \begin{array}{lllllll} & 2 & 3 & 4 & 5 & 6\end{array}$

21. To understand who I am as a person, you have $\quad \begin{array}{llllllll}1 & 2 & 3 & 4 & 5 & 6\end{array}$ to know that I'm LGB.

22. I get very confused when I try to figure out my $\quad \begin{array}{lllllll}1 & 2 & 3 & 4 & 5 & 6\end{array}$ sexual orientation.

23. I have felt comfortable with my sexual identity $\quad \begin{array}{lllllll}1 & 2 & 3 & 4 & 5 & 6\end{array}$ just about from the start.

24. Being an LGB person is a very important aspect $\begin{array}{lllllll}1 & 2 & 3 & 4 & 5 & 6\end{array}$ of my life.

25. I believe being LGB is an important part of me. $\begin{array}{lllllll}1 & 2 & 3 & 4 & 5 & 6\end{array}$

26. I am proud to be LGB. $\quad \begin{array}{lllllll}1 & 2 & 3 & 4 & 5 & 6\end{array}$

27. I believe it is unfair that I am attracted to people $\begin{array}{lllllll}1 & 2 & 3 & 4 & 5 & 6\end{array}$ of the same sex. 


\section{APPENDIX F}

\section{Internal Review Board Approval (Initial)}

IRB protocol number: 1305043671

Title: The Roles of Resilience and Perceived Discrimination in Sexual Minority Identity Development and Integration

\section{PI: Monica Leppma}

The West Virginia University Institutional Review Board approved the above-referenced protocol on 15-Nov-2013. To access this protocol, click on the protocol number link provided. Your approval letter can be found in the History subsection of the Summary \& History section located on the Protocol Actions page. For more information, see the Viewing Correspondence quick reference guide. Any future protocol action requests can be completed through the WVU+kc system.

Questions related to NHSR, Full Board, Emergency Use, Clinical Trials, or CIRB protocols as well as amendments, renewals, deviations/violations/exceptions, or adverse events/UPIRTSOs should be directed to Lilo Ast at 304.293.7555 or lilo.ast@mail.wvu.edu.

Questions related to Exempt or Expedited protocols should be directed to Barbara White at 304.293.5971 or barb.white@mail.wvu.edu.

Training or troubleshooting questions should be directed to Jonathan Young at 304.293.1119 or jonathan.young@mail.wvu.edu. 


\section{APPENDIX G}

\section{Internal Review Board Approval (Amendment)}

IRB protocol number: 1305043671A001

Title: The Roles of Resilience and Perceived Discrimination in Sexual Minority Identity Development and Integration

\section{PI: Monica Leppma}

The West Virginia University Institutional Review Board approved the above-referenced protocol on 20-Dec-2013. To access this protocol, click on the protocol number link provided. Your approval letter can be found in the History subsection of the Summary \& History section located on the Protocol Actions page. For more information, see the Viewing Correspondence quick reference guide. Any future protocol action requests can be completed through the WVU+kc system.

Questions related to NHSR, Full Board, Emergency Use, Clinical Trials, or CIRB protocols as well as amendments, renewals, deviations/violations/exceptions, or adverse events/UPIRTSOs should be directed to Lilo Ast at 304.293.7555 or lilo.ast@mail.wvu.edu.

Questions related to Exempt or Expedited protocols should be directed to Barbara White at 304.293.5971 or barb.white@mail.wvu.edu.

Training or troubleshooting questions should be directed to Jonathan Young at 304.293.1119 or jonathan.young@mail.wvu.edu. 


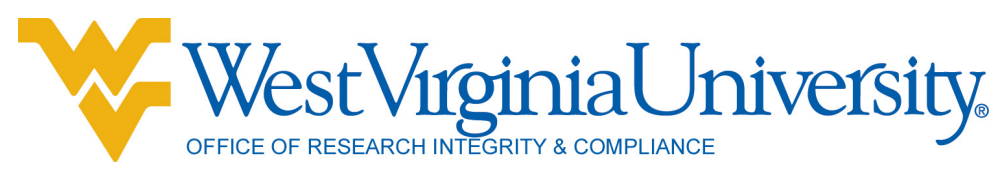

\author{
Human Research Protocol \\ Only Minimal Risk Consent Form \\ Without HIPAA
}

\title{
Only Minimal Risk \\ Consent Information Form (without HIPAA)
}

\author{
Principal Investigator Monica Leppma, Ph.D. \\ Department \\ Protocol Number \\ Study Title \\ Counseling, Rehabilitation Counseling, and Counseling Psychology \\ 1305043671 \\ Minority Identity Development and Integration \\ Co-Investigator(s) \\ Sponsor (if any) \\ Peter Alexander Brown, M.A. \\ N/A
}

\section{Contact Persons}

Research at West Virginia University involving human participants is carried out under the oversight of the Institutional Review Board (WVU IRB). This research has been reviewed and approved by the IRB. In the event you experience any side effects or injury related to this research, you should contact Dr. Monica Leppma at (304) 293-0540. (After hours contact: Alexander Brown at (718) 869-1480). If you have any questions, concerns, or complaints about this research, you can contact Dr. Monica Leppma at (304) 293-0540 or Alexander Brown at (718) 869-1480.

For information regarding your rights as a research subject, to discuss problems, concerns, or suggestions related to the research, to obtain information or offer input about the research, contact the Office of Research Integrity \& Compliance at (304) 293-7073.

In addition if you would like to discuss problems, concerns, have suggestions related to research, or would like to offer input about the research, contact the Office of Research Integrity and Compliance at 304-293-7073.

\section{Introduction}

You have been asked to participate in this research study, which has been explained to you by Alexander Brown. This study is being conducted by Monica Leppma, Ph.D. in the Department of Counseling, Rehabilitation Counseling, and Counseling Psychology at West Virginia University with funding provided by the College of Education and Human Services.

\section{Purpose(s) of the Study}

The purpose of the present study is to better understand the occurrence of sexual minority identity integration across social roles as predicted by such protective factors as resilience. It has been well understood in the literature for some time now that public disclosure of one's identity is an inadequate and unrealistic developmental marker, given the risk of discrimination or rejection often associated with coming out. In order to draw conclusions about the facilitative effects of resilience of sexual minority identity, the purpose of this study will be to (a) determine whether resilience factors and perception of discrimination are predictive of identity integration, and (b) evaluate the relationship between integration and dimensions of sexual minority identity development. 


\section{Description of Procedures}

This study involves completing this anonymous online survey. You will be asked to fill out a questionnaire regarding your sexual orientation. This will take approximately 15 minutes. You do not have to answer all the questions. You will have the opportunity to see the questionnaire before signing this consent form.

\section{Discomforts}

There are no known or expected risks from participating in this study, except for the mild frustration associated with answering the questions.

\section{Benefits}

You may not receive any direct benefit from this study. The knowledge gained from this study may eventually benefit others.

\section{Financial Considerations}

There are no special fees for participating in this study. You will not be paid for participating. If you intend to participate in the optional raffle, you will be asked to provide your name and address for the purposes of contacting winners. Identifying information, however, will not be connected to survey responses. Therefore, disclosed personal information cannot be linked to individual participants.

\section{Confidentiality}

Any information about you that is obtained as a result of your participation in this research will be kept as confidential as legally possible. Your research records and test results, just like hospital records, may be subpoenaed by court order or may be inspected by the study sponsor or federal regulatory authorities (including the FDA if applicable) without your additional consent.

In addition, there are certain instances where the researcher is legally required to give information to the appropriate authorities. These would include mandatory reporting of infectious diseases, mandatory reporting of information about behavior that is imminently dangerous to your child or to others, such as suicide, child abuse, etc.

In any publications that result from this research, neither your name nor any information from which you might be identified will be published without your consent.

\section{Voluntary Participation}

Participation in this study is voluntary. You are free to withdraw your consent to participate in this study at any time.

Refusal to participate or withdrawal will not affect [your class standing or grades, as appropriate] and will involve no penalty to you. Refusal to participate or withdrawal will not affect your future care, or your employee status at West Virginia University.

In the event new information becomes available that may affect your willingness to participate in this study, this information will be given to you so that you can make an informed decision about whether or not to continue your participation.

You have been given the opportunity to ask questions about the research, and you have received answers concerning areas you did not understand.

I willingly agree to be in the study.
$\circ$ Yes
$\circ$ No 